\title{
Symmetry, complexity and multicritical point of the two-dimensional spin glass
}

\author{
Jean-Marie Maillard $\dagger$, Koji Nemoto $\ddagger$ and Hidetoshi Nishimori§ \\ $\nmid$ †PTHE, Tour 24, 5 ème étage, case 7109, 2 Place Jussieu, 75251 Paris Cedex 05, \\ France \\ $\ddagger$ Division of Physics, Hokkaido University, Sapporo 060-0810, Japan \\ $\S$ Department of Physics, Tokyo Institute of Technology, Oh-okayama, Meguro-ku, \\ Tokyo 152-8551, Japan
}

\begin{abstract}
We analyze models of spin glasses on the two-dimensional square lattice by exploiting symmetry arguments. The replicated partition functions of the Ising and related spin glasses are shown to have many remarkable symmetry properties as functions of the edge Boltzmann factors. It is shown that the applications of homogeneous and Hadamard inverses to the edge Boltzmann matrix indicate reduced complexities when the elements of the matrix satisfy certain conditions, suggesting that the system has special simplicities under such conditions. Using these duality and symmetry arguments we present a conjecture on the exact location of the multicritical point in the phase diagram.

PACS numbers: $05.50 .+\mathrm{q}, 75.10 . \mathrm{Hk}, 75.50 . \mathrm{Lk}$
\end{abstract}

\section{Introduction}

Properties of spin glasses are well understood in mean-field models, which show such anomalous behaviour as replica symmetry breaking, multivalley structure, and slow dynamics [1, 2]. The difficult problem of whether or not these mean-field predictions apply to realistic finite-dimensional systems is still largely unsolved, and active investigations are carried out mainly using numerical methods 2]. Very few systematic analytical work exists, an exception being a symmetry argument using gauge invariance to derive the exact internal energy and several other exact/rigorous relations [3, 4.

In the present paper we develop another type of symmetry argument for models of spin glasses in two dimensions under the replica formalism. Our analyses reveal a variety of invariance properties of the replicated partition function under transformations of the edge Boltzmann factors, a notable example of which is the duality transformation.

Also discussed are complexities of the edge Boltzmann matrix under inversions. It is well established that integrable systems such as the standard scalar Potts model have remarkably reduced complexities of the edge Boltzmann matrix when the parameters satisfy integrability conditions [5]. Analogous (but not the same) behaviour is observed 
in our present problem, suggesting simplified (if not integrable) properties of the systems in restricted regions in the phase diagram.

The results concerning symmetry properties are used to present a conjecture on the location of the multicritical point in the phase diagram. Although the argument is not a mathematically complete proof, we expect the prediction to be exact for several reasons including agreement with numerical results in many different models and satisfaction of inequalities.

It should be remembered that most of the analyses are for real replicas, that is, the number of replicas is a positive integer. The quenched limit of vanishing number of replicas is discussed in relation only to limited cases including the conjectured location of the multicritical point.

In the next section symmetries of the system are derived. The replicated partition function is demonstrated to be invariant under several different types of transformations of the edge Boltzmann factors. It is shown that a special subvariety exists which satisfies remarkable enhanced symmetries. Complexities under applications of matrix inverses are treated in section 3. Here also the same subvariety as above is seen to have reduced complexities, suggesting its special role. A conjecture on the multicritical point is presented in section 4 and numerical evidence supporting the conjecture is discussed. The final section is devoted to summary and discussions.

\section{Symmetries of the partition function}

In this section we first derive the expression of the replicated partition function in terms of the edge Boltzmann factors. Then the symmetries and related properties of the partition function are discussed. The arguments are first developed for the $\pm J$ Ising model and then are generalized to a broader class of models (such as the Gaussian spin glass and random chiral Potts model) in the last part of this section.

\subsection{Replicated partition function}

Let us start by considering the Hamiltonian of the $\pm J$ Ising model

$$
H=-\sum_{\langle i j\rangle} J_{i j} S_{i} S_{j},
$$

where $J_{i j}$ is ferromagnetic $J(>0)$ with probability $p$ and antiferromagnetic $-J$ with $1-p$. The sum extends over nearest neighbours on the square lattice. The randomness in $J_{i j}$ will be treated by the replica method. We will mainly consider the case of positive integer $n$, the number of replicas. Some results for the quenched limit $n \rightarrow 0$ will be discussed in subsequent sections. The constraints of Ising spins and the $\pm J$ distribution of the interactions will be relaxed later in the present section. 
Symmetry, complexity and multicritical point of the two-dimensional spin glass

After the average over bond configurations, the system becomes spatially homogeneoust, and the partition function

$$
\left[Z^{n}\right]_{\mathrm{av}} \equiv Z_{n}
$$

where the square brackets with subscript 'av' denote the configurational average, is specified uniquely by the edge Boltzmann matrix representing neighbouring interactions. For example, when $n=1$ (the annealed model), the edge Boltzmann matrix is

$$
p \cdot\left[\begin{array}{ll}
w_{0} & w_{1} \\
w_{1} & w_{0}
\end{array}\right]+(1-p) \cdot\left[\begin{array}{ll}
w_{1} & w_{0} \\
w_{0} & w_{1}
\end{array}\right] \equiv\left[\begin{array}{cc}
x_{0} & x_{1} \\
x_{1} & x_{0}
\end{array}\right] \equiv A_{1},
$$

where $w_{0}=e^{K}, w_{1}=e^{-K}$ with $K=J / k_{B} T$, and $x_{0}$ denotes the edge Boltzmann factor for parallel configuration of neighbouring spins whereas $x_{1}$ is for antiparallel spins. Similarly, for $n=2$ we have the edge Boltzmann matrix

$$
p \cdot\left[\begin{array}{ll}
w_{0} & w_{1} \\
w_{1} & w_{0}
\end{array}\right] \otimes\left[\begin{array}{ll}
w_{0} & w_{1} \\
w_{1} & w_{0}
\end{array}\right]+(1-p) \cdot\left[\begin{array}{ll}
w_{1} & w_{0} \\
w_{0} & w_{1}
\end{array}\right] \otimes\left[\begin{array}{ll}
w_{1} & w_{0} \\
w_{0} & w_{1}
\end{array}\right] .
$$

This is a $4 \times 4$ matrix of hierarchical structure

$$
\left[\begin{array}{cccc}
x_{0} & x_{1} & x_{1} & x_{2} \\
x_{1} & x_{0} & x_{2} & x_{1} \\
x_{1} & x_{2} & x_{0} & x_{1} \\
x_{2} & x_{1} & x_{1} & x_{0}
\end{array}\right] \equiv A_{2}=\left[\begin{array}{cc}
A_{1} & B_{1} \\
B_{1} & A_{1}
\end{array}\right]
$$

with $x_{0}=p w_{0}^{2}+(1-p) w_{1}^{2}, x_{1}=p w_{0} w_{1}+(1-p) w_{1} w_{0}$ and $x_{2}=p w_{1}^{2}+(1-p) w_{0}^{2}$. The $2 \times 2$ matrix $B_{1}$ is obtained from $A_{1}$ by replacing $x_{0}$ and $x_{1}$ with $x_{1}$ and $x_{2}$, respectively: $B_{1}=A_{1}\left(x_{0} \rightarrow x_{1}, x_{1} \rightarrow x_{2}\right)$. The element $x_{0}$ represents the two parallel spin pairs on neighbouring sites (such as ++ for replica 1 and ++ for replica 2 ), $x_{1}$ is for one parallel and one antiparallel pairs (like,+++- ), and $x_{2}$ corresponds to two antiparallel pairs $(+-,-+$ for example).

The edge Boltzmann matrix of larger $n$ can be derived by recursions. The general formula is

$$
p \cdot\left[\begin{array}{cc}
w_{0} & w_{1} \\
w_{1} & w_{0}
\end{array}\right]^{\otimes n}+(1-p) \cdot\left[\begin{array}{cc}
w_{1} & w_{0} \\
w_{0} & w_{1}
\end{array}\right]^{\otimes n} \equiv A_{n}=\left[\begin{array}{cc}
A_{n-1} & B_{n-1} \\
B_{n-1} & A_{n-1}
\end{array}\right],
$$

where $B_{n-1}=A_{n-1}\left(x_{k} \rightarrow x_{k+1} ; k=0,1, \cdots, n-1\right)$. The partition function (2) is a function of the matrix elements of $A_{n}$,

$$
Z_{n}\left(x_{0}, x_{1}, \cdots, x_{n}\right) .
$$

Here $x_{k}$ denotes the Boltzmann factor of the spin configuration with $k$ antiparallel spin pairs among $n$ neighbouring pairs.

$\ddagger$ except for boundary effects which are irrelevant to thermodynamic properties and will be ignored 
In the symmetry arguments developed subsequently, we will often consider the case where the elements $x_{k}$ are independent of each other although they are originally related through the parameters of the $\pm J$ Ising model $(K$ and $p$ ) by the relations

$$
\begin{aligned}
x_{0}= & p w_{0}^{n}+(1-p) w_{1}^{n} \\
x_{1}= & p w_{0}^{n-1} w_{1}+(1-p) w_{1}^{n-1} w_{0} \\
x_{2}= & p w_{0}^{n-2} w_{1}^{2}+(1-p) w_{1}^{n-2} w_{0}^{2} \\
& \vdots \\
x_{n}= & p w_{1}^{n}+(1-p) w_{0}^{n} .
\end{aligned}
$$

An advantage to consider generic points in the space spanned by the elements of the edge Boltzmann matrix

$$
\mathcal{S}=\left\{\boldsymbol{x}=\left(x_{0}, x_{1}, x_{2}, \cdots, x_{n}\right) \mid x_{k} \in \mathbb{R}(k=0,1, \cdots, n)\right\}
$$

is that we can discuss various well-known models such as the $2^{n}$-state standard scalar Potts model which has $x_{1}=x_{2}=x_{3}=\cdots=x_{n}$ : All but one $\left(x_{0}\right)$ spin configurations have the same Boltzmann factors. The $n$-replicated $\pm J$ Ising model with the edge Boltzmann factor (8) lies on a two-dimensional submanifold $\mathcal{T}$ of $\mathcal{S}$ :

$$
\mathcal{T}=\left\{\boldsymbol{x} \in \mathcal{S} \mid x_{k}=p w_{0}^{n-k} w_{1}^{k}+(1-p) w_{1}^{n-k} w_{0}^{k}(k=0,1, \cdots, n)\right\}
$$

\subsection{Duality}

Some spin systems in two dimensions have invariance properties under duality transformations. The formulation of duality by $\mathrm{Wu}$ and Wang [6] is particularly useful for our problem having the edge Boltzmann matrix (6) of hierarchical structure. According to these authors, the dual Boltzmann factors are derived simply by Fourier sums applied to each $2 \times 2$ block (corresponding to each replica) of the edge Boltzmann matrix (6) $\oint$. The simplest case is the annealed model $n=1$ of equation (3): Its dual Boltzmann factors are the sum and difference of the original Boltzmann factors with appropriate normalization,

$$
\begin{aligned}
& \sqrt{2} x_{0}^{*}=x_{0}+x_{1} \\
& \sqrt{2} x_{1}^{*}=x_{0}-x_{1} .
\end{aligned}
$$

As an example, when the system is purely ferromagnetic $p=1$ in equation (3), if we define the dual coupling $K^{*}$ by $e^{-2 K^{*}}=x_{1}^{*} / x_{0}^{*}$ (remembering $e^{-2 K}=x_{1} / x_{0}=w_{1} / w_{0}$ ), we have from equation (11) the familiar duality relation of the ferromagnetic Ising model,

$$
e^{-2 K^{*}}=\frac{w_{0}-w_{1}}{w_{0}+w_{1}}=\tanh K
$$

In the case of $n=2$ with equations (41) and (5), it is necessary to generate combinations of sums and differences of appropriate matrix elements to obtain the dual Boltzmann factors,

$$
\begin{aligned}
& 2 x_{0}^{*}=\left(x_{0}+x_{1}\right)+\left(x_{1}+x_{2}\right)=x_{0}+2 x_{1}+x_{2} \\
& 2 x_{1}^{*}=\left(x_{0}-x_{1}\right)+\left(x_{1}-x_{2}\right)=x_{0}-x_{2} \\
& 2 x_{2}^{*}=\left(x_{0}-x_{1}\right)-\left(x_{1}-x_{2}\right)=x_{0}-2 x_{1}+x_{2} .
\end{aligned}
$$

$\S$ Fourier sums for the two-component (Ising) case are just the sum and difference of two elements. 
Symmetry, complexity and multicritical point of the two-dimensional spin glass

The formula for general $n$ with the edge Boltzmann matrix (6) is

$$
\Delta_{n}=\frac{1}{2^{n}}\left[\begin{array}{cc}
1 & 1 \\
1 & -1
\end{array}\right]^{\otimes n} \cdot\left[\begin{array}{cc}
A_{n-1} & B_{n-1} \\
B_{n-1} & A_{n-1}
\end{array}\right] \cdot\left[\begin{array}{cc}
1 & 1 \\
1 & -1
\end{array}\right]^{\otimes n},
$$

where the entries of the diagonal matrix $\Delta_{n}$ are the dual Boltzmann factors $2^{n / 2} x_{m}^{*}$ in a certain order. More explicitly, the dual Boltzmann factors, which are linear combinations of the original Boltzmann factors, can be written from equation (14) as

$$
2^{n / 2} x_{m}^{*}=\sum_{k=0}^{n} D_{m}^{k} x_{k}
$$

where the $D_{m}^{k}$ are the coefficients of the expansion of $(1-t)^{m} \cdot(1+t)^{n-m}$ :

$$
(1-t)^{m} \cdot(1+t)^{n-m}=\sum_{k=0}^{n} D_{m}^{k} \cdot t^{k}
$$

that is,

$$
D_{m}^{k}=\sum_{l=0}^{k}(-1)^{l}\left(\begin{array}{c}
m \\
l
\end{array}\right)\left(\begin{array}{c}
n-m \\
k-l
\end{array}\right) .
$$

To understand equation (16) we first note that $m$ antiparallel pairs are chosen to generate $x_{m}^{*}$ for dual spin pairs, which is reflected in the parameter $m$ on the left-hand side of this equation. This is equivalent to choosing $m$ of the second rows $(1,-1)$ from the direct product of $n 2 \times 2$ matrices in equation (14) $\|$. Then we choose $k$ antiparallel pairs of original spins as in equation (17) to obtain the coefficient of $x_{k}$.

On the square lattice the partition function remains invariant under the duality transformation of edge Boltzmann factors [6]:

$$
Z_{n}\left(x_{0}, x_{1}, x_{2}, \cdots, x_{n}\right)=Z_{n}\left(x_{0}^{*}, x_{1}^{*}, x_{2}^{*}, \cdots, x_{n}^{*}\right) \quad(\boldsymbol{x} \in \mathcal{S}),
$$

apart from a trivial factor $2^{n}$ and boundary effects, both of which are irrelevant to thermodynamic properties and will be ignored in this paper. Note that the symmetry under duality (18) is valid for any values of the edge Boltzmann factors $x_{0}, x_{1}, x_{2}, \cdots, x_{n}$, which do not necessarily satisfy the relation (8) of the $\pm J$ Ising model as indicated by the symbol $\boldsymbol{x} \in \mathcal{S}$ at the end of equation (18).

It will be useful to write explicitly the general duality transformation (15) applied to the specific case of the $\pm J$ Ising model for later use, namely, for the case $\boldsymbol{x} \in \mathcal{T}(\subset \mathcal{S})$. Again we first write the formula for the simple case of $n=2$ written in equation (13) so that the reader understands the structure:

$$
\begin{aligned}
2 x_{0}^{*} & =p\left(w_{0}+w_{1}\right)^{2}+(1-p)\left(w_{1}+w_{0}\right)^{2}=\left(w_{0}+w_{1}\right)^{2} \\
2 x_{1}^{*} & =p\left(w_{0}+w_{1}\right)\left(w_{0}-w_{1}\right)+(1-p)\left(w_{1}+w_{0}\right)\left(w_{1}-w_{0}\right) \\
& =(2 p-1)\left(w_{0}+w_{1}\right)\left(w_{0}-w_{1}\right) \\
2 x_{2}^{*} & =p\left(w_{0}-w_{1}\right)^{2}+(1-p)\left(w_{1}-w_{0}\right)^{2}=\left(w_{0}-w_{1}\right)^{2} .
\end{aligned}
$$

$\|$ As seen in equation (11), an antiparallel spin pair in the dual space corresponds to the difference (the row $(1,-1))$ of two original Boltzmann factors. 
The general formula is

$$
\begin{aligned}
2^{n / 2} x_{2 m}^{*} & =\left(w_{0}+w_{1}\right)^{n-2 m}\left(w_{0}-w_{1}\right)^{2 m} \\
2^{n / 2} x_{2 m+1}^{*} & =(2 p-1)\left(w_{0}+w_{1}\right)^{n-2 m-1}\left(w_{0}-w_{1}\right)^{2 m+1}
\end{aligned}
$$

\subsection{Symmetries under sign changes of coupling constants}

In the present subsection the edge Boltzmann factors are regarded as functions of the parameters of the $\pm J$ Ising model following equation (8), $\boldsymbol{x} \in \mathcal{T}$. The partition function $Z_{n}$ is invariant under the change of the sign of coupling constant $K \rightarrow-K$ at all bonds if the system is on the square lattice. This change of the sign exchanges $w_{0}$ and $w_{1}$, and consequently, according to equation (8), $x_{k}$ is exchanged with $x_{n-k}$ :

$$
Z_{n}\left(x_{0}, x_{1}, x_{2}, \cdots, x_{n}\right)=Z_{n}\left(x_{n}, x_{n-1}, x_{n-2}, \cdots, x_{0}\right) \quad(\boldsymbol{x} \in \mathcal{T}) .
$$

Combination of two symmetries, duality (18) and change of sign of $K$ (21), leads to another symmetry. On the right-hand side of equation (16) the change of sign of $t$ is equivalent to the change of the sign of $D_{m}^{k}$ for odd $k$. The left-hand side of equation (16) suggests that the change of sign of $t$ is also realized by exchange of $m$ and $n-m$. Then

we conclude in equation (15) that the change of sign of $x_{k}$ for odd $k$ on the right-hand side

$$
D_{m}^{k} x_{k} \rightarrow(-1)^{k} D_{m}^{k} x_{k}
$$

should be performed simultaneously with the exchange of $x_{m}^{*}$ and $x_{n-m}^{*}$ to keep this duality equation valid. Using duality (18) we therefore find the following symmetry,

$$
\begin{aligned}
& Z_{n}\left(x_{0},-x_{1}, x_{2},-x_{3}, \cdots,(-1)^{n} x_{n}\right) \\
= & Z_{n}\left(x_{n}^{*}, x_{n-1}^{*}, \cdots, x_{1}^{*}, x_{0}^{*}\right) \\
= & Z_{n}\left(x_{0}^{*}, x_{1}^{*}, \cdots, x_{n-1}^{*}, x_{n}^{*}\right) \\
= & Z_{n}\left(x_{0}, x_{1}, \cdots, x_{n}\right) \quad(\boldsymbol{x} \in \mathcal{T}) .
\end{aligned}
$$

The second equality comes from equation (21) and the final relation is duality (18).

When the number of replicas is even $n=2 q$, the partition function has additional symmetry which exchanges $x_{k}$ with $x_{2 q-k}$ for $k$ odd only:

$$
\begin{aligned}
& Z_{2 q}\left(x_{0}, x_{1}, x_{2}, x_{3}, x_{4}, \cdots, x_{2 q-3}, x_{2 q-2}, x_{2 q-1}, x_{2 q}\right) \\
= & Z_{2 q}\left(x_{0}, x_{2 q-1}, x_{2}, x_{2 q-3}, x_{4}, \cdots, x_{3}, x_{2 q-2}, x_{1}, x_{2 q}\right) \quad(\boldsymbol{x} \in \mathcal{T}) .
\end{aligned}
$$

It is convenient to start the proof from the following expression of the edge Boltzmann factors generalizing equation (8),

$$
\begin{aligned}
x_{0} & =\sum_{l} p_{l} e^{2 q K_{l}} \\
x_{1} & =\sum_{l} p_{l} e^{2(q-1) K_{l}} \\
x_{2} & =\sum_{l} p_{l} e^{2(q-2) K_{l}}
\end{aligned}
$$


Symmetry, complexity and multicritical point of the two-dimensional spin glass

$$
\begin{aligned}
& \vdots \\
x_{2 q-2} & =\sum_{l} p_{l} e^{-2(q-2) K_{l}} \\
x_{2 q-1} & =\sum_{l} p_{l} e^{-2(q-1) K_{l}} \\
x_{2 q}= & \sum_{l} p_{l} e^{-2 q K_{l}},
\end{aligned}
$$

where the sum runs over $l=1,2$ with $p_{1}=p, p_{2}=1-p$ and $K_{1}=K, K_{2}=-K$ for the $\pm J$ Ising model. We would like the $x_{k}$ with $k$ even to remain invariant and the $x_{k}$ with $k$ odd to be changed into $x_{2 q-k}$. As one can see from equation (25), this may be considered to be a transformation $K \rightarrow-K$ for odd $k$ only:

$$
x_{k}(K) \rightarrow x_{k}\left((-1)^{k} \cdot K\right) .
$$

The sign $(-1)^{k}$ actually corresponds to the sign of $\Sigma_{i} \cdot \Sigma_{j}$ for neighbouring sites $i$ and $j$, where $\Sigma_{i}=\sigma_{i}^{(1)} \sigma_{i}^{(2)} \cdots \sigma_{i}^{(2 q)}$ and $\Sigma_{j}=\sigma_{j}^{(1)} \sigma_{j}^{(2)} \cdots \sigma_{j}^{(2 q)}$, because $\Sigma_{i} \cdot \Sigma_{j}$ is 1 for even number of antiparallel pairs and is -1 otherwise. The formula (25) can be written as

$$
x_{k}=\sum_{l} p_{l} \exp \left(K_{l} \sum_{\alpha=1}^{n} \sigma_{i}^{(\alpha)} \sigma_{j}^{(\alpha)}\right)
$$

where $k$ pairs among $2 q$ pairs of neighbouring spins are antiparallel $\sum_{\alpha} \sigma_{i}^{(\alpha)} \sigma_{j}^{(\alpha)}=$ $2 q-2 k$. Changing $K_{l}$ as

$$
K_{l} \rightarrow \Sigma_{i} \cdot \Sigma_{j} \cdot K_{l}
$$

amounts to changing the edge Boltzmann factor as follows :

$$
x_{k} \rightarrow \sum_{l} p_{l} \exp \left(K_{l} \cdot \Sigma_{i} \cdot \Sigma_{j} \sum_{\alpha=1}^{n} \sigma_{i}^{(\alpha)} \sigma_{j}^{(\alpha)}\right) .
$$

In other words this is equivalent to performing for each replica a non-trivial Mattis transformation depending on all the other replicas:

$$
\sigma_{i}^{(\alpha)} \rightarrow \tau_{i}^{(\alpha)}=\Sigma_{i} \cdot \sigma_{i}^{(\alpha)}=\prod_{\beta \neq \alpha} \sigma_{i}^{(\beta)} .
$$

Note that, as far as dummy variables to be summed on are concerned, the $\tau_{i}^{(\alpha)}$ 's are as good as the $\sigma_{i}^{(\alpha)}$ 's : This is a one-to-one change of variables. Actually one goes back from the $\tau_{i}^{(\alpha)}$ 's to the $\sigma_{i}^{(\alpha)}$ 's by performing the same transformation as the one defining the $\tau_{i}^{(\alpha)}$, s :

$$
\tau_{i}^{(\alpha)} \rightarrow \sigma_{i}^{(\alpha)}=\prod_{\beta \neq \alpha} \tau_{i}^{(\beta)}=\prod_{\beta \neq \alpha}\left(\Sigma_{i} \cdot \sigma_{i}^{(\beta)}\right)=\Sigma_{i}^{2 q-1} \cdot \Sigma_{i} \cdot \sigma_{i}^{(\alpha)} .
$$

We have used the identity $\Sigma_{i}^{2 q}=+1$. Therefore this Mattis transformation is an involution, and we have proved equation (26) which is equivalent to the symmetry (24).

Let us point out another interesting symmetry of the partition function which can be derived from a combination of symmetries discussed so far. If we denote the duality 
transformation (15) symbolically as $D$ and the exchange of $x_{k}$ and $x_{2 q-k}$ for even $n$ and odd $k$ in equation (24) as $M$, the combination

$$
D_{2}=D \cdot M
$$

also leaves the partition function invariant as long as the system satisfies the conditions for $D$ and $M$ to be the true symmetry such as the lattice structure (square lattice) and even $n$. This duality-like symmetry under the transformation $D_{2}$ will be used in the next subsection. It is worth noticing here that $D$ and $M$ commute for $n$ even $(D \cdot M=M \cdot D)$, and $D, M$ and $D_{2}$ are all involutions, $D^{2}=M^{2}=D_{2}^{2}=\mathbf{1}$.

\subsection{Subvariety and duality}

The $\pm J$ Ising model with quenched randomness has remarkable properties along a line (curve) in the phase diagram defined by the relation

$$
\exp (-2 K)=\frac{1-p}{p}
$$

known as the Nishimori line (NL) [3, 4. Similar interesting behaviour is observed on the same line also in the replicated system with $n=2$ [7]. In this subsection we analyze symmetries of the replicated system with general integer $n$ under the condition (33) using the results of previous subsections.

Let us consider the $\pm J$ Ising model on the square lattice whose parameters satisfy equation (33). From equations (18) and (33), the latter being equivalent to $p=w_{0} /\left(w_{0}+w_{1}\right)$ and $1-p=w_{1} /\left(w_{0}+w_{1}\right)$, it is straightforward to see that the following relations hold:

$$
x_{1}=x_{n}, x_{2}=x_{n-1}, x_{3}=x_{n-2}, \cdots, x_{k}=x_{n-k+1}, \cdots .
$$

The condition (33) reduces the degree of freedom from two $(p$ and $K)$ to one. This means that we restrict ourselves to a one-dimensional curve in the $(n+1)$-dimensional

space $\mathcal{S}$. It will be useful to relax this constraint and consider the $([(n+1) / 2]+1)$ dimensional submanifold of $\mathcal{S}$ specified only by equation (34), where $[x]$ stands for the largest integer not exceeding $x$. We shall call this submanifold the subvariety $\mathcal{N}$ :

$$
\mathcal{N}=\left\{\boldsymbol{x} \in \mathcal{S} \mid x_{k}=x_{n-k+1}(k=1,2, \cdots, n)\right\} .
$$

The subvariety $\mathcal{N}$ of course includes the one-dimensional curve NL specified by equation (33): NL $\subset \mathcal{N}$.

By the duality (15), $\mathcal{N}$ is transformed into the dual $\mathcal{N}^{*}$ satisfying

$$
x_{1}=x_{2}, x_{3}=x_{4}, x_{5}=x_{6}, \cdots, x_{2 m-1}=x_{2 m}, \cdots .
$$

To prove this fact, we first note that the condition (34) implies that the coefficient of $x_{k}\left(=x_{n-k+1}\right)$ on the right-hand side of equation (15) is

$$
D_{m}^{k}(n)+D_{m}^{n-k+1}(n),
$$


where we have written the $n$-dependence of $D_{m}^{k}$ explicitly. Then the relation (36) follows if we can show

$$
D_{2 m-1}^{k}(n)+D_{2 m-1}^{n-k+1}(n)=D_{2 m}^{k}(n)+D_{2 m}^{n-k+1}(n),
$$

the left-hand side of which is the coefficient of $x_{k}\left(=x_{n-k+1}\right)$ of $2^{n / 2} x_{2 m-1}^{*}$ in equation (15) and the right-hand side is for $x_{k}\left(=x_{n-k+1}\right)$ of $2^{n / 2} x_{2 m}^{*}$. Equation (38) can be proved by induction with respect to $n$ : The validity for small $n(=1,2,3)$ is checked trivially. Let us assume that that equation (38) is valid for $n$. Then it is not difficult to show that the same equation holds for $n+1$ using the following recursion relation

$$
D_{m}^{k}(n+1)=D_{m}^{k}(n)+D_{m}^{k-1}(n),
$$

which is derived by multiplying both sides of equation (16) by $1+t$ (which amounts to the change $n \rightarrow n+1)$. This ends the proof.

Comparison of equations (34) and (36) suggests that the subvariety $\mathcal{N}$ is in general not self-dual, $\mathcal{N} \neq \mathcal{N}^{*}$. When $n$ is even $n=2 q$, the partition function has an additional symmetry (24), $M$ in the notation of equation (32). Then the combined transformation $D_{2}=D \cdot M$ keeps the subvariety invariant, $\mathcal{N}=\mathcal{N}^{*}$ : The application of $D_{2}$ to $\mathcal{N}$ is shown to yield the same relation as equation (34)

$$
x_{1}^{*}=x_{2 q}^{*}, x_{2}^{*}=x_{2 q-1}^{*}, x_{3}^{*}=x_{2 q-2}^{*}, \cdots, x_{m}^{*}=x_{2 q-m+1}^{*}, \cdots .
$$

The proof is outlined in Appendix A. We therefore conclude that the subvariety $\mathcal{N}$ is globally self-dual when $n$ is even, that is, any point in the submanifold defined by equation (34) is mapped by $D_{2}$ to another point in the same submanifold (but is not fixed point-by-point in general).

\subsection{Inversions}

The edge Boltzmann matrix $A_{n}$ with generic values of the elements $\left(x_{0}, x_{1}, x_{2}, \cdots, x_{n}\right)$ has a remarkable property that its inverse matrix has the same structure; the inverse matrix has the same arrangement of elements as the original matrix. To show this we first note that the entries of the Boltzmann matrix are defined up to a common multiplicative factor, and therefore we can also discuss, instead of the matrix inversion $A_{n} \rightarrow A_{n}^{-1}$, a homogeneous matrix inversion transformation $I$ (actually a homogeneous polynomial transformation):

$$
A_{n} \rightarrow A_{n}^{-1} \cdot \operatorname{det}\left(A_{n}\right) .
$$

Let us also introduce the homogeneous transformation $J$ corresponding to the inversion of elements of the dual matrix (Hadamard inverse), $y_{k} \rightarrow 1 / y_{k}(k=0,1, \cdots, n) \boldsymbol{\Phi}$,

$$
J: \quad y_{k} \longrightarrow \prod_{m=0, m \neq k}^{m=n} y_{m} .
$$

At first sight, performing the matrix inversion of the $2^{n} \times 2^{n}$ Boltzmann matrix $A_{n}$ may seem to yield quite large calculations. However, since the duality transformation

ๆ Transformation $J$ is analogous to negating various coupling constants. 
actually diagonalizes matrix $A_{n}$ as was mentioned in relation to equation (14), it is straightforward to see that the matrix inversion just amounts to changing the dual variables $x_{m}^{*}$ into their simple inverse : $x_{m}^{*} \rightarrow 1 / x_{m}^{*}$. From this remark, it is straightforward to see that the matrix inverse of $A_{n}$ is a $2^{n} \times 2^{n}$ matrix of the same form as the original $A_{n}$ (but of course with different elements : $x_{k} \rightarrow x_{k}^{\prime}$ ). With obvious notations, and since there is no possible confusion, we will also denote by $I$ this transformation on the $x_{k}$ 's :

$$
I: \quad x_{k} \quad \longrightarrow \quad x_{k}^{\prime}
$$

Up to a common multiplicative factor one thus has (since $D$ is an involution, that is, $\left.D^{2}=1\right)$ :

$$
D \cdot I \cdot D \propto J, \quad \text { or } \quad I \propto D \cdot J \cdot D .
$$

Remark : One should recall that the two involutions $I$ and $J$ are actually (non-linear) symmetries of the phase diagrams of (anisotropic) spin edge lattice models [5, 8, 9]. From equation (44) one sees that these "non-linear" symmetries of the phase diagrams are closely related to the (linear) duality symmetry. A duality symmetry exists when the edge Boltzmann matrix corresponds to cyclic matrices or semi-direct product of cyclic matrices [6]. However, when a duality symmetry does not exist, like for instance the Ising model in a magnetic field, the two "non-linear" symmetries $I$ and $J$ still exist and can still be used to analyse the phase diagram [8].

\subsection{Spin representation of the dual Boltzmann factor}

The elements of the dual edge Boltzmann factors of the replicated $\pm J$ Ising model (20) have an interesting symmetry. It is instructive to take the ratios of $x_{1}^{*}, x_{2}^{*}, \cdots$ to $x_{0}^{*}$ (which is equivalent to setting the energy level of all-parallel state to 0 ):

$$
\begin{aligned}
x_{2 m-1}^{*} / x_{0}^{*} & =(2 p-1)\left(\frac{w_{0}-w_{1}}{w_{0}+w_{1}}\right)^{2 m-1}=(2 p-1) \tanh ^{2 m-1} K \\
x_{2 m}^{*} / x_{0}^{*} & =\tanh ^{2 m} K .
\end{aligned}
$$

It is observed that the right-hand side is multiplied by tanh $K$ each time the number of antiparallel spin pairs $m$ increases. Another factor $2 p-1$ appears alternately. Therefore the right-hand side of equation (45) can be expressed as a simple Boltzmann factor of the dual system,

$$
A \exp \left\{K^{*}\left(S^{(1)}+S^{(2)}+\cdots+S^{(n)}\right)+K_{p}^{*} S^{(1)} S^{(2)} \cdots S^{(n)}\right\},
$$

where $S^{(\alpha)}$ is the product of neighbouring dual spins in the $\alpha$ th replica $\left(S^{(\alpha)}=S_{i}^{(\alpha)} S_{j}^{(\alpha)}\right)$, and $K^{*}$ and $K_{p}^{*}$ are the dual couplings corresponding to thermal and randomness parameters:

$$
\tanh K=e^{-2 K *}, 2 p-1=e^{-2 K_{p}^{*}} .
$$

This expression (46) has a very interesting interpretation. The first part can be interpreted as being driven by thermal fluctuations since it has only the (dual) thermal 
coupling $K^{*}$ in front of the spin variables. This first term is decoupled explicitly from the second part which is understood to be driven by quenched randomness as it is controlled by the (dual) coupling $K_{p}^{*}$ determined only by $p$. The first term causes ferromagnetic ordering of dual spin variables whereas the second term enhances spin-glass-like multireplica ordering.

The condition (33) is written in terms of the dual couplings as $K^{*}=K_{p}^{*}$. Therefore, on the NL, the above-mentioned two types of ordering tendency exactly balance. This reminds us of the result that the ferromagnetic ordering dominates above the NL whereas spin-glass order is larger below [10]. The balance $K^{*}=K_{p}^{*}$ may also be called enhanced symmetry because the single-replica and the multi-replica terms have exactly the same coupling.

It should be pointed out that a larger type of enhanced symmetry is realized by the $2^{n}$-state standard scalar Potts model which has the edge Boltzmann factor

$$
\begin{aligned}
& A \exp \left\{K_{1}\left(S^{(1)}+S^{(2)}+\cdots+S^{(n)}\right)\right. \\
& +K_{2}\left(S^{(1)} S^{(2)}+S^{(1)} S^{(3)}+\cdots+S^{(n-1)} S^{(n)}\right) \\
& \left.+\cdots+K_{n} S^{(1)} S^{(2)} \cdots S^{(n)}\right\}
\end{aligned}
$$

with all couplings equal $K_{1}=K_{2}=K_{3}=\cdots=K_{n}$ : It is straightforward to confirm that this Boltzmann factor has just two values, one for $S^{(1)}=S^{(2)}=\cdots=S^{(n)}=1$ and the other for all other configurations, thus representing the standard scalar Potts model.

These two models, the $\pm J$ Ising model (46) on the NL (with $K^{*}=K_{p}^{*}$ ) and the $2^{n}$ state standard scalar Potts model (48) (with $K_{1}=K_{2}=K_{3}=\cdots=K_{n}$ ), coincide when $n=2$ but not in general. We may therefore regard the $n$-replicated $\pm J$ Ising model on the NL as a system with similar but a little weaker symmetry than the standard scalar Potts model with $2^{n}$ states. Another point to notice is that the original Boltzmann factors (8) of the $\pm J$ Ising model are not expressible in a simple form like equation (46): If we try to do so by adjusting the coupling constants in equation (48), each $K_{i}$ will appear with a different value from the other coefficients. Only the dual system has the simple form of the edge Boltzmann factor $\left(K_{2}=K_{3}=\cdots=K_{n-1}=0\right)$.

\subsection{Results derived from the dual Boltzmann factor}

The effective edge Boltzmann factor (46) shows that the replicated $\pm J$ Ising model in the dual representation has a relatively simple Hamiltonian with positive ferromagnetic couplings. This fact enables us to analyze the model using known results on ferromagnetic spin systems. One of our interests to be discussed later will be the structure of the phase diagram of the $n$-replicated system. The phase diagram drawn in terms of the original parameters $p$ and $T$ should look topologically the same as the one with axes $1 / K_{p}^{*}$ and $K^{*}$ because $1 / K_{p}^{*}$ is a monotone increasing function of $p$ and $K^{*}$ is also monotonic in $T$ (see figure 1). Note that in the present paper we call the point marked in black dot in figure 1 the multicritical point irrespective of the order of 
transition. Of course one should take into account that ordered and disordered phases are exchanged between the original and dual systems.

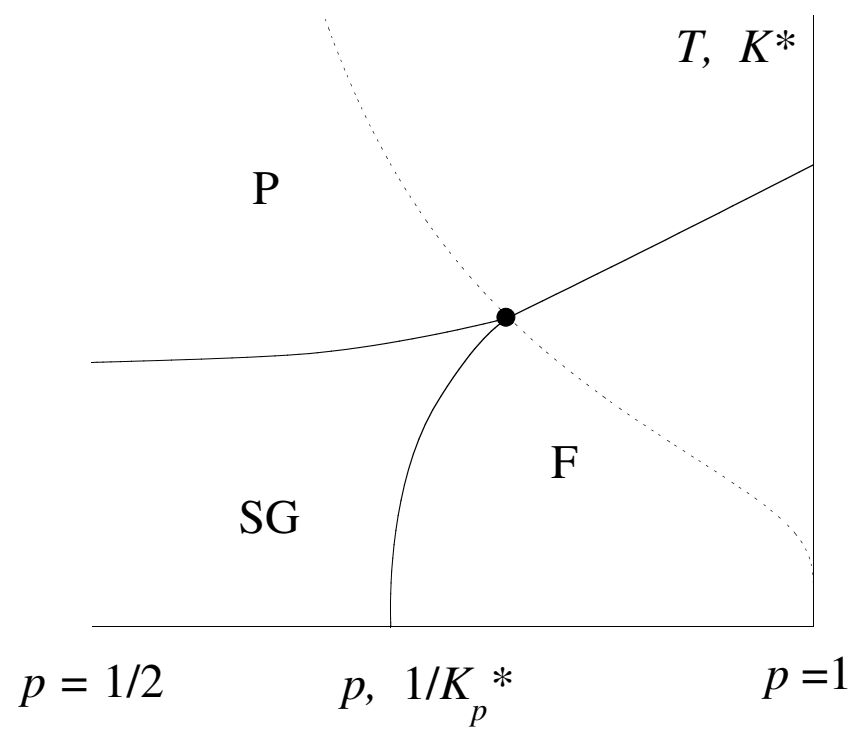

Figure 1. Generic phase diagram of the $n$-replicated Ising model. The multicritical point (black dot) lies on the NL (shown in a thin dotted curve).

We first check a few limiting cases. According to the dual Boltzmann factor (46), when $K_{p}^{*} \rightarrow 0(p \rightarrow 1)$, the system decouples into $n$ independent ferromagnetic Ising models as expected. If, on the other hand, $K^{*} \rightarrow 0$ (or the low-temperature limit $T \rightarrow 0$ in the original variable), only the multi-replica coupling $K_{p}^{*}$ survives in equation (46). By redefining spins as $\sigma_{i}=S_{i}^{(1)} S_{i}^{(2)} \cdots S_{i}^{(n)}$, the system reduces to a simple ferromagnetic Ising model. The critical point exists at $K_{p}^{*}=K_{\mathrm{c}}^{\mathrm{F}}$, where $K_{\mathrm{c}}^{\mathrm{F}}$ is the critical point of the ferromagnetic Ising model satisfying $e^{-2 K_{\mathrm{c}}^{\mathrm{F}}}=\sqrt{2}-1$. Therefore the original system in the ground state has a critical point at $p_{\mathrm{c}}=\frac{\sqrt{2}}{2}=0.7071$ (derived from $e^{-2 K_{p}^{*}}=\sqrt{2}-1$ ) irrespective of $n$ as long as $n$ is a positive integer. Note that the quenched limit $n \rightarrow 0$ is expected to have a different critical probability $p_{\mathrm{c}}$ near 0.89 (see the next section). This observation means that the two limits $n \rightarrow 0$ and $T \rightarrow 0$ do not commute.

The other limit $K_{p}^{*} \rightarrow \infty$ corresponds to $p=\frac{1}{2}$ in the original variable. According to equation (46), the dual spins are then constrained as $S^{(1)} S^{(2)} \cdots S^{(n)}=1$. It follows that the $n$th spin variable can be expressed as $S^{(n)}=S^{(1)} S^{(2)} \cdots S^{(n-1)}$. Then the dual Boltzmann factor becomes

$$
A \exp \left\{K^{*}\left(S^{(1)}+S^{(2)}+\cdots+S^{(n-1)}+S^{(1)} S^{(2)} \cdots S^{(n-1)}\right)\right\} .
$$

This is exactly the Boltzmann factor of the $(n-1)$-replicated system with $K_{p}^{*}=K^{*}$. We have therefore established the following relation for the partition functions of the $n$ and $(n-1)$-replicated $\pm J$ Ising models:

$$
Z_{n}\left(K, K_{p}=0\right) \propto Z_{n-1}\left(K, K_{p}=K\right),
$$


where the partition function is expressed by using the original couplings ( $K_{p}$ defined by $e^{-2 K_{p}^{*}}=\tanh K_{p}$ is the dual of $K_{p}^{*}$ ). The trivial constants in front of the partition function are omitted. The identity (150) proves that the $n$-replicated system with $p=\frac{1}{2}\left(K_{p}=0\right)$ is equivalent to the $(n-1)$-replicated system on the NL, $K=K_{p}$ (equivalent to $K^{*}=K_{p}^{*}$ ).

The effective edge Boltzmann factor (46) represents a system with ferromagnetic couplings only. Thus the Griffiths inequalities hold [11. In particular, first derivatives of an arbitrary correlation function are positive semi-definite:

$$
\frac{\partial}{\partial K^{*}}\left\langle S_{i}^{(\alpha)} S_{j}^{(\beta)} S_{k}^{(\gamma)} \cdots\right\rangle \geq 0, \quad \frac{\partial}{\partial K_{p}^{*}}\left\langle S_{i}^{(\alpha)} S_{j}^{(\beta)} S_{k}^{(\gamma)} \cdots\right\rangle \geq 0
$$

These inequalities imply that the boundaries between ferromagnetic and nonferromagnetic phases are monotonic in $K^{*}$ and $K_{p}^{*}$ (and in the original variables $T$ and $p$ ) as drawn schematically in figure1. The reason is that, otherwise, the system will show reentrant behaviour and consequently the order parameters will be non-monotonic, violating (151).

An interesting consequence of monotonic behaviour of the phase boundaries is that the transition temperature at $p=\frac{1}{2}$ between the paramagnetic and spin glass phases (to be denoted as $\left.T_{\mathrm{c}}^{(n)}\left(\frac{1}{2}\right)\right)$ is smaller than or equal to the temperature of the multicritical point $T_{\mathrm{c}}^{(n)}(\mathrm{MCP})$ which lies generically on the NL. Since we have already established in equation (501) that the $n$-replicated system on the line $p=\frac{1}{2}$ is equivalent to the $(n-1)$-replicated system on the NL, it is concluded that

$$
T_{\mathrm{c}}^{(n-1)}(\mathrm{MCP})=T_{\mathrm{c}}^{(n)}\left(\frac{1}{2}\right) \leq T_{\mathrm{c}}^{(n)}(\mathrm{MCP}) .
$$

In terms of $p$, this inequality reads, using $K=K_{p}(\tanh K=2 p-1)$ satisfied by the multicritical point to rewrite $T$ by $p$,

$$
p_{\mathrm{c}}^{(n-1)}(\mathrm{MCP}) \geq p_{\mathrm{c}}^{(n)}(\mathrm{MCP}) .
$$

The value of $p$ at the multicritical point is a monotone decreasing function of $n$. We can then derive a lower bound for $p_{\mathrm{c}}^{(0)}(\mathrm{MCP})$ of the quenched system $n \rightarrow 0$ if we remember that the $n=1$ annealed system is easily solved by reducing it to the regular ferromagnetic Ising model. The result is

$$
p_{\mathrm{c}}^{(0)}(\mathrm{MCP}) \geq \frac{1+\sqrt{\sqrt{2}-1}}{2}=0.821797 \ldots
$$

Although this bound is not very tight since the expected value of the left-hand side is approximately 0.89 as discussed in the next section, it is nevertheless useful to have a mathematically rigorous bound (if we accept that the $n \rightarrow 0$ limit causes no problems).

We can also derive an inequality

$$
T_{\mathrm{c}}^{(n)}\left(\frac{1}{2}\right) \leq T_{\mathrm{c}}^{(n+1)}\left(\frac{1}{2}\right)
$$

from equation (52). 
Another non-trivial fact is the absence of reentrant behaviour as mentioned already. It is well established for $n=1$ and $n=2$ that the boundary between the ferromagnetic and non-ferromagnetic phases below the multicritical point is never reentrant as depicted in figure 1in accordance with the present rigorous result [7]. It is a subtle matter whether or not this monotonicity still holds in the quenched limit $n \rightarrow 0$ : If this is the case, the phase boundary below the multicritical point should be a vertical straight line in accordance with the argument in 12 because the absence of ferromagnetic phase to the left of the multicritical point at any temperature is rigorously established in the quenched model [3]. However we avoid to make a definite statement here since it is not obvious that monotonicity derived from inequalities proved for positive integer $n$ remains valid in the limit $n \rightarrow 0$.

\subsection{Relations between $n$ and $n+1$}

It has been established in equation (50) that the $n$-replicated system with $p=\frac{1}{2}$ is equivalent to $(n-1)$-replicated system on the NL on the square lattice. This result has already been pointed out by Georges et al [7]. We here derive several further relations on physical quantities of $n$ - and $(n+1)$-replicated systems. The arguments in the present subsection apply to the replicated $\pm J$ Ising model on an arbitrary lattice in an arbitrary dimension. No duality will be used.

It is instructive to rederive the relation between the partition functions $Z_{n}$ and $Z_{n+1}$ without recourse to duality. The randomness average of the $n$-replicated partition function of the $\pm J$ Ising model is written as [3, 4]

$$
Z_{n}\left(K, K_{p}\right)=\frac{1}{\left(2 \cosh K_{p}\right)^{N_{B}}} \sum_{\tau} e^{K_{p} \sum \tau_{i j}} Z(K)^{n},
$$

where $N_{B}$ is the number of bonds of the lattice, $\tau_{i j}= \pm 1$ is the sign of $J_{i j}(= \pm J)$, and $Z(K)$ is the partition function with fixed randomness. After gauge transformation and summation over gauge variables, the exponential in the above expression turns to a partition function with coupling $K_{p}$ [3, 4]:

$$
Z_{n}\left(K, K_{p}\right)=\frac{1}{2^{N}\left(2 \cosh K_{p}\right)^{N_{B}}} \sum_{\tau} Z\left(K_{p}\right) Z(K)^{n},
$$

where $N$ is the total number of spins. It readily follows from this equation that $Z_{n}(K, K)$ and $Z_{n+1}(K, 0)$ are essentially equal to each other:

$$
2^{N}(2 \cosh K)^{N_{B}} Z_{n}(K, K)=2^{N+N_{B}} Z_{n+1}(K, 0) .
$$

This is the identity (50) we already derived using duality for the square lattice. The present result is more general as we did not use the properties of a specific lattice.

Similar identities hold for order parameters. The magnetization is defined by

$$
m_{n}\left(K, K_{p}\right)=\frac{\sum_{\tau} e^{K_{p} \sum \tau_{i j}}\left(\sum_{S} S_{i} e^{-\beta H(S)}\right) Z(K)^{n-1}}{\sum_{\tau} e^{K_{p} \sum \tau_{i j}} Z(K)^{n}}
$$


where $\beta=1 / k_{B} T$. Spins on boundaries are fixed to up states to avoid trivial vanishing of the single-spin expectation value due to global inversion symmetry. Gauge transformation brings this equation into

$$
m_{n}\left(K, K_{p}\right)=\frac{\sum_{\tau}\left(\sum_{\sigma} \sigma_{i} e^{K_{p} \sum \tau_{i j} \sigma_{i} \sigma_{j}}\right)\left(\sum_{S} S_{i} e^{-\beta H(S)}\right) Z(K)^{n-1}}{\sum_{\tau} Z\left(K_{p}\right) Z(K)^{n}} .
$$

This expression of magnetization is to be compared with the spin glass order parameter, which can be written using gauge transformation as

$$
\begin{aligned}
q_{n}\left(K, K_{p}\right) & =\frac{\sum_{\tau} e^{K_{p} \sum \tau_{i j}}\left(\sum_{S} S_{i} e^{-\beta H(S)}\right)^{2} Z(K)^{n-2}}{\sum_{\tau} e^{K_{p} \sum \tau_{i j}} Z(K)^{n}} \\
& =\frac{\sum_{\tau}\left(\sum_{S} S_{i} e^{-\beta H(S)}\right)^{2} Z\left(K_{p}\right) Z(K)^{n-2}}{\sum_{\tau} Z\left(K_{p}\right) Z(K)^{n}} .
\end{aligned}
$$

Comparison of equations (60) and (61) immediately leads to

$$
m_{n}(K, K)=q_{n}(K, K)=q_{n+1}(K, 0) .
$$

The first equality shows that there is no spin glass phase on the NL if we define the spin glass phase by $m_{n}=0$ and $q_{n}>0$. The second equality of (62) indicates that the spin glass order parameter of the $n$-replicated system on the NL is exactly equal to that of the $(n+1)$-replicated system with $p=\frac{1}{2}$.

The above identity (62) on the order parameters can be generalized to a relation between the distribution functions of order parameters [4]. We define and gaugetransform the distribution function of magnetization as

$$
\begin{aligned}
& P_{m}^{(n)}\left(x ; K, K_{p}\right) \\
& =\frac{\sum_{\tau} e^{K_{p} \sum \tau_{i j}}\left(\sum_{S} \delta\left(x-\frac{1}{N} \sum_{i} S_{i}\right) e^{-\beta H(S)}\right) Z(K)^{n-1}}{\sum_{\tau} e^{K_{p} \sum \tau_{i j} Z(K)^{n}}} \\
& =\frac{\sum_{\tau}\left(\sum_{S} \sum_{\sigma} \delta\left(x-\frac{1}{N} \sum_{i} S_{i} \sigma_{i}\right) e^{-\beta H(S)} e^{-\beta_{p} H(\sigma)}\right) Z(K)^{n-1}}{\sum_{\tau} Z\left(K_{p}\right) Z(K)^{n}},
\end{aligned}
$$

where $\beta_{p} H(\sigma)$ is the Hamiltonian with coupling $K_{p}$ and spin variables $\sigma$. The distribution of spin glass order parameter, measuring the overlap of two replicas, is treated similarly. Using gauge transformation it is written as

$$
\begin{aligned}
& P_{q}^{(n)}\left(x ; K, K_{p}\right) \\
& =\frac{\sum_{\tau} e^{K_{p} \sum \tau_{i j}}\left(\sum_{S} \sum_{\sigma} \delta\left(x-\frac{1}{N} \sum_{i} S_{i} \sigma_{i}\right) e^{-\beta H(S)} e^{-\beta H(\sigma)}\right) Z(K)^{n-2}}{\sum_{\tau} e^{K_{p} \sum \tau_{i j} Z(K)^{n}}} \\
& =\frac{\sum_{\tau}\left(\sum_{S} \sum_{\sigma} \delta\left(x-\frac{1}{N} \sum_{i} S_{i} \sigma_{i}\right) e^{-\beta H(S)} e^{-\beta H(\sigma)}\right) Z\left(K_{p}\right) Z(K)^{n-2}}{\sum_{\tau} Z\left(K_{p}\right) Z(K)^{n}}
\end{aligned}
$$

It is easy to see from equations (63) and (64) that the following relation holds

$$
P_{m}^{(n)}(x ; K, K)=P_{q}^{(n)}(x ; K, K)=P_{q}^{(n+1)}(x ; K, 0) .
$$

Since the distribution function of a single-replica variable like magnetization $P_{m}(x)$ has only a trivial structure (with at most two delta peaks), it follows from the first equality 
of (65) that the distribution of the spin glass order parameter $P_{q}(x)$ is also trivial on the NL for any $n$ : There exists no complex structure such as replica-symmetry breaking. The second equality of (65) then proves that the same trivial structure holds for the spin-glass distribution function of $(n+1)$-replicated system with $p=\frac{1}{2}$. Thus the spin glass phase of the $(n+1)$-replicated system with $p=\frac{1}{2}$ has only a trivial structure. We should be careful when we consider the possibility to apply this result to the distribution

function $P_{q}^{(n+1)}$ of the quenched system which, in the present context, corresponds to $n+1 \rightarrow 0$ or $n \rightarrow-1$. The arguments in the present subsection are mathematically rigorous only for positive integer $n$.

\subsection{Generalization}

Many of the arguments so far have been on the replicated $\pm J$ Ising model although some of the symmetry relations are valid in more general systems with generic edge Boltzmann matrix with hierarchical structure. In the present subsection we apply the duality relations to the random Ising model with general coupling values as well as to the random $Z_{q}$ model which includes the random chiral Potts model.

Let us first treat the $n$-replicated Ising model with a set of couplings $K_{1}, K_{2}, K_{3}, \cdots$ with respective probabilities $p_{1}, p_{2}, p_{3}, \cdots$. The sign of $K_{j}$ is arbitrary in this subsection: All the couplings can be ferromagnetic, for example. The hierarchical structure of the edge Boltzmann matrix is the same as the one already discussed for the $\pm J$ Ising model.

The edge Boltzmann factors are

$$
\begin{gathered}
x_{0}=\sum_{j} p_{j} e^{n K_{j}} \\
x_{1}=\sum_{j} p_{j} e^{(n-2) K_{j}} \\
x_{2}=\sum_{j} p_{j} e^{(n-4) K_{j}} \\
\vdots \\
x_{n}=\sum_{j} p_{j} e^{-n K_{j}} .
\end{gathered}
$$

The dual are then

$$
\begin{aligned}
2^{n / 2} x_{0}^{*} & =\sum_{j} p_{j}\left(e^{K_{j}}+e^{-K_{j}}\right)^{n} \\
2^{n / 2} x_{1}^{*} & =\sum_{j} p_{j}\left(e^{K_{j}}+e^{-K_{j}}\right)^{n-1}\left(e^{K_{j}}-e^{-K_{j}}\right) \\
2^{n / 2} x_{2}^{*} & =\sum_{j} p_{j}\left(e^{K_{j}}+e^{-K_{j}}\right)^{n-2}\left(e^{K_{j}}-e^{-K_{j}}\right)^{2} \\
& \vdots \\
2^{n / 2} x_{n}^{*} & =\sum_{j} p_{j}\left(e^{K_{j}}-e^{-K_{j}}\right)^{n},
\end{aligned}
$$

a generalization of equations (19) and (20). It is sometimes convenient to take the continuum limit of randomness distribution. The original and dual Boltzmann factors are then

$$
x_{k} \quad=\int d u P(u) e^{(n-2 k) \beta u}
$$


Symmetry, complexity and multicritical point of the two-dimensional spin glass

$$
2^{n / 2} x_{m}^{*}=\int d u P(u)\left(e^{\beta u}+e^{-\beta u}\right)^{n-m}\left(e^{\beta u}-e^{-\beta u}\right)^{m} .
$$

A generalization of equation (33) is expressed as the probability distribution of the form [3, 4]

$$
P(u)=e^{\beta u} F\left(u^{2}\right),
$$

where $F\left(u^{2}\right)$ is an arbitrary even function of $u$ and should be chosen to satisfy the normalization condition of $P(u)$. The constraint (171) applied to the edge Boltzmann factors (69) and (70) gives the symmetry properties

$$
\begin{aligned}
& x_{1}=x_{n}, \quad x_{2}=x_{n-1}, \quad x_{3}=x_{n-2}, \cdots \\
& x_{1}^{*}=x_{2}^{*}, \quad x_{3}^{*}=x_{4}^{*}, \quad x_{5}^{*}=x_{6}^{*}, \cdots .
\end{aligned}
$$

The first equation (72) can be checked directly from equations (69) and (71). The second (73) has already been proved generically in equation (36). When $n$ is even, a further symmetry exists as in equation (40), implying that the subvariety $\mathcal{N}$ is globally self-dual, $\mathcal{N}=\mathcal{N}^{*}$.

We next turn to the random chiral $Z_{q}$ model for which again we use the $\mathrm{Wu}-$ Wang duality. The arguments in the previous subsections do not apply directly because we have used a binary (Ising) structure of basic variables. Each spin variable is now assumed to have $q$ components to be symbolized by integers from 0 to $q-1$. The interaction will be written as $V(k)$ when the difference in the neighbouring spin variables is $k$. The Ising model has $q=2$, and the $q$-state standard scalar Potts model satisfies $V(1)=V(2)=\cdots=V(q-1)$. The interaction is cyclic, $V(k+q)=V(k)$. Chiral randomness is assumed to exist: The interaction energy for the difference in neighbouring spin variables being $k$ is changed from the non-random value $V(k)$ to $V(k+l)$ with probability $p_{l}$.

The $k$ th edge Boltzmann factor of the original lattice is

$$
x_{k}=\sum_{l=0}^{q-1} p_{l} e^{n V(l+k)} .
$$

The dual Boltzmann factors are obtained by Fourier transform of the edge Boltzmann matrix, a $q$-state generalization of equation (14), and we only write the result here. See Appendix B for some details.

$$
\begin{aligned}
q^{n / 2} x_{0}^{*} & =\sum_{l} p_{l}\left(\sum_{k=0}^{q-1} e^{V(k+l)}\right)^{n} \\
q^{n / 2} x_{1}^{*} & =\sum_{l} p_{l}\left(\sum_{k} e^{V(k+l)}\right)^{n-1}\left(\sum_{k} \omega^{k} e^{V(k+l)}\right) \\
q^{n / 2} x_{2}^{*} & =\sum_{l} p_{l}\left(\sum_{k} e^{V(k+l)}\right)^{n-1}\left(\sum_{k} \omega^{2 k} e^{V(k+l)}\right)
\end{aligned}
$$


Symmetry, complexity and multicritical point of the two-dimensional spin glass

$$
\begin{aligned}
& q^{n / 2} x_{q-1}^{*}=\sum_{l} p_{l}\left(\sum_{k} e^{V(k+l)}\right)^{n-1}\left(\sum_{k} \omega^{(q-1) k} e^{V(k+l)}\right) \\
& q^{n / 2} x_{(q-1)+1}^{*}=\sum_{l} p_{l}\left(\sum_{k} e^{V(k+l)}\right)^{n-2}\left(\sum_{k} \omega^{k} e^{V(k+l)}\right)^{2}
\end{aligned}
$$

where $\omega$ is the $q$ th root of unity. These expressions, in particular those for $x_{0}$ and $x_{0}^{*}$, will be used in later sections.

\section{Complexity generated by inversions}

Since $I$ and $J$ are two (non-linear) symmetries of the phase diagram of the (anisotropic) model [5, 8, 9], it is natural to combine these two involutions considering the infinite order (birational) transformation $K=I \cdot J$. Keeping in mind that $I$ is basically $D \cdot J \cdot D$ as in equation (44), we find that the iteration of $K=I \cdot J$ amounts to iterating $k=D \cdot J$.

The transformation $K$ is generically an infinite order (birational) transformation which is a canonical symmetry of the parameter space of the model and an infinite discrete symmetry of the Yang-Baxter equations (star-triangle relations for spin-edge models) when the model is integrable [9]. This amounts to studying the iteration of transformation $K$ and, in particular, the complexity of this transformation [13, namely the growth of the degrees [14] of the (numerator or denominator of the) successive rational expressions encountered in the iteration ${ }^{+}$. Generically one gets an exponential growth of the calculations like $\lambda^{L}$ with the number $L$ of iterations. We will call from now on $\lambda$ the complexity of the transformation $K$ or the complexity of the associated spin edge lattice model. In this "complexity" framework, an enhanced symmetry, occurring for some submanifold (in fact an algebraic subvariety) of the parameter space of the model (here the $x_{k}$ 's), corresponds to a reduction of the complexity $\lambda$ to a smaller value: the integrable subcases pop out as algebraic subvarieties associated with a (at most) polynomial growth of the calculations [5, 9] $(\lambda=1)$.

Let us first remark that the subvariety $\mathcal{N}$ is clearly (globally) invariant by $J$. The duality $D$ permutes $\mathcal{N}$ with its dual subvariety $\mathcal{N}^{*}$. The dual subvariety $\mathcal{N}^{*}$ (see equation (36) $)$ is also clearly (globally) invariant by $J$. Keeping in mind that $I$ is basically $D \cdot J \cdot D$, one deduces immediately that $\mathcal{N}$ is also (globally) invariant by $I$, and is, thus, is (globally) invariant by $K=I \cdot J\left(\mathcal{N}\right.$ is (globally) invariant by $k^{2}$ where $k=D \cdot J)$.

+ Or the purely numerical growth rate of the number of digits of the (numerators or denominators of the) successive rational numbers obtained when iterating an initial rational point [5]. Since the entries of the matrices grow exponentially, one has to use special representations of the integers allowing to manipulate large values [15] 
We have calculated the "complexities" $\lambda$ corresponding to the iteration of $k=$ $D \cdot J$, for a general $2^{n} \times 2^{n}$ cyclic matrix, then for the general $2^{n} \times 2^{n}$ matrix (6) for various number of replicas $n$ and, finally, for Boltzmann factors restricted to the subvariety $\mathcal{N}$ of the $2^{n} \times 2^{n}$ matrices ([6) . The results are summarized in the following table :

\begin{tabular}{|c|c|c|c|c|}
\hline$n$ & $n=2$ & $n=3$ & $n=4$ & $n=5$ \\
\hline Cyclic & 1 & 5.8284 & 13.9282 & 29.9666 \\
\hline Matrix (6) & 1 & 2 & 3.2143 & 4.2361 \\
\hline $\mathcal{N}$ & 1 & 1.4142 & 1.6180 & 2.3344 \\
\hline
\end{tabular}

These values are all related to the solutions of polynomial equations with integer coefficients: They are the inverse of the smallest root of various polynomials with integer coefficients. For instance the complexities of a general $2^{n} \times 2^{n}$ cyclic matrix, displayed in the first row of this table, correspond to the smallest root of polynomial $1-\left(2^{n}-2\right) t+t^{2}$ for various values of $n$. The complexities for generic $2^{n} \times 2^{n}$ matrices of the form (6), associated with $n$ replicas, correspond respectively to $1-t, 1-2 t, 1-3 t-t^{2}+t^{3}$, and $1-4 t-t^{2}$. Finally, the complexities for $2^{n} \times 2^{n}$ matrices of the form (6) restricted to the subvariety $\mathcal{N}$, respectively correspond to $1-t, 1-2 t^{2}, 1-t-t^{2}$, and $1-6 t^{2}+3 t^{4}$.

We have also obtained the complexities $\lambda_{K}$ associated with the iteration of $K=$ $I \cdot J$, instead of $k=D \cdot J$. These $\lambda_{K}$ 's are (as it should, since $K$ is equivalent to $k^{2}$ ) the square of the $\lambda^{\prime}$ 's displayed in the previous table. For a general $2^{n} \times 2^{n}$ non-cyclic matrix for which one does not have a duality anymore, one, surprisingly, finds, up to the accuracy of our calculations, the same complexities $\lambda_{K}$ as for a cyclic $2^{n} \times 2^{n}$ matrix! The $2^{n} \times 2^{n}$ matrices of the form (6) are, of course, subcases of the general non-cyclic $2^{n} \times 2^{n}$ matrices. One thus finds quite a drastic complexity reduction $\left((5.82842)^{2} \rightarrow 2^{2},(13.9282)^{2} \rightarrow(3.214319)^{2}, \ldots\right)$ when restricting $2^{n} \times 2^{n}$ matrices to the form (6) corresponding to effective Boltzmann matrix for $n$ replicas. The replica analysis thus defines naturally a class of highly remarkable (non-random) lattice models, namely (6), which are very interesting per se. We also see very clearly, that condition $\mathcal{N}$ defines a highly singled-out subvariety of the previous remarkable models for which further drastic reductions of complexity occur (enhanced symmetry).

It would be tempting to restrict models (6) to various (global or point-by-point) selfdual conditions (like $x_{0}^{*}=x_{0}, x_{1}^{*}=x_{1}, \ldots$ ) in order to see similar drastic reductions of complexities, and possibly a polynomial growth of complexity on some integrable subvariety, or on some critical submanifold* (possibly given by intersections of the subvariety $\mathcal{N}$ and various self-dual conditions) of the $x_{0}, \cdots x_{n}$ parameter space $\mathcal{S}$. Such an analysis, in fact, requires, on a square lattice for instance, to generalize our isotropic effective model (6) to an anisotropic effective model with two different sets of horizontal and vertical Boltzmann matrices both of the form (6). The calculations

* including the cases of first order transitions 
become much more subtle and involved and, thus, will be detailed elsewhere. Let us briefly say that our preliminary "complexity analysis" results are not in disagreement with the numerical results displayed below.

\section{Conjecture on the location of the multicritical point}

We have discussed symmetry and complexity of replicated random spin systems and their generalizations. We now apply the results to the presentation of a conjecture on the exact location of the multicritical point in the phase diagram.

\subsection{General structure and conjecture}

In the homogeneous variables $x_{k}$, the duality relation we have derived for the $\pm J$ Ising model is (in the homogeneous partition function)

$$
Z_{n}\left(x_{0}, x_{1}, \cdots, x_{n}\right)=Z_{n}\left(x_{0}^{*}, x_{1}^{*}, \cdots, x_{n}^{*}\right) .
$$

The question we try to answer in the present section is whether or not it is possible to identify the transition point with a fixed point of duality transformation for the replicated $\pm J$ Ising model and its generalizations (the Gaussian Ising model and random $Z_{q}$ model) as is the case for the non-random ferromagnetic Ising model and the standard scalar Potts model on the square lattice. Mathematically rigorous argument on this problem can be developed only in a few limited cases. We nevertheless take a step further to present a conjecture that the fixed-point condition $x_{0}=x_{0}^{*}$ of only one of the $n+1$ independent variables has the possibility to lead to the exact transition point if we restrict ourselves to the one-dimensional subvariety with enhanced symmetry, the NL. Numerical data support our conjecture.

Let us first consider the generic case of arbitrary values of $x_{0}, x_{1}, x_{2}, \cdots, x_{n}$, not just on the NL, until otherwise stated. It is convenient to extract the factor $x_{0}$, the edge Boltzmann factor for all-parallel spin state, in front of the partition function in equation (80):

$$
Z_{n}\left(x_{0}, x_{1}, x_{2}, \cdots, x_{n}\right) \equiv\left(x_{0}\right)^{N_{B}} \mathcal{Z}_{n}\left(u_{1}, u_{2}, \cdots, u_{n}\right),
$$

where $N_{B}$ is the number of bonds on the lattice, and the reduced variables are defined by $u_{k}=x_{k} / x_{0}$. This representation implies that we measure the energy relative to the all-parallel (perfectly ferromagnetic) state. The duality relation (80) is rewritten in terms of $\mathcal{Z}_{n}$ as

$$
\left(x_{0}\right)^{N_{B}} \mathcal{Z}_{n}\left(u_{1}, u_{2}, \cdots, u_{n}\right)=\left(x_{0}^{*}\right)^{N_{B}} \mathcal{Z}_{n}\left(u_{1}^{*}, u_{2}^{*}, \cdots, u_{n}^{*}\right),
$$

where $u_{k}^{*}=x_{k}^{*} / x_{0}^{*}$.

When $n=1$, this form of duality allows us to identify the fixed point with the transition point because the partition function has only one variable $\mathcal{Z}\left(u_{1}\right)$, and $u_{1}^{*}$ is a monotone decreasing function of $u_{1}$ : The transition point, if unique, should be at the fixed point $u_{1}=u_{1}^{*}$. It then follows from equation (82) that $x_{0}$ also satisfies the fixed 
point condition $x_{0}=x_{0}^{*}$ because the functional values of $\mathcal{Z}_{n}$ on both sides are equal when the arguments $u_{1}$ and $u_{1}^{*}$ coincide. We can therefore use the fixed point condition $x_{0}=x_{0}^{*}$ in place of $u_{1}=u_{1}^{*}$ to locate the transition point. If we apply this argument to the $\pm J$ Ising model (the annealed model), the condition $x_{0}=x_{0}^{*}$ defines a curve which is the exact phase boundary in the $p-T$ phase diagram as depicted in figure 2 .

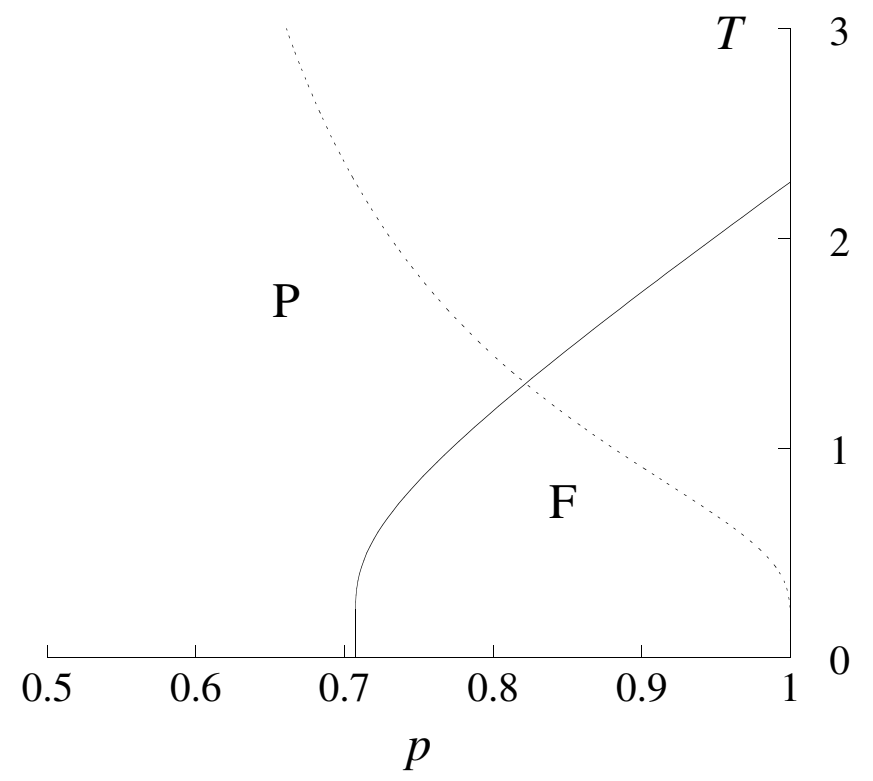

Figure 2. Phase diagram of the $\pm J$ Ising model with $n=1$. The phase boundary is given by $x_{0}=x_{0}^{*}$ and the NL is shown dotted.

For $n=2$, the situation is not very different. The condition $x_{0}=x_{0}^{*}$ is equivalent to $x_{1}=x_{1}^{*}$ as well as to $x_{2}=x_{2}^{*}$ as can be verified from equation (13). Thus we have $u_{1}=u_{1}^{*}$ and $u_{2}=u_{2}^{*}$ as soon as we impose the fixed point condition of the principal edge Boltzmann factor, $x_{0}=x_{0}^{*}$. This fact is of course consistent with equation (82) because both the prefactor $\left(x_{0}\right)^{N_{B}}$ and the functional value $\mathcal{Z}_{n}\left(u_{1}, u_{2}\right)$ are the same on both sides when $x_{0}=x_{0}^{*}$. In the case of the $\pm J$ Ising model, this fixed point condition $x_{0}=x_{0}^{*}$ defines a curve in the phase diagram drawn in figure 3 (full curve above the multicritical point and dashed curve terminating at $p=0.5$ ). As discussed by Georges et al [7], this curve coincides with the exact phase boundary above the multicritical point on the NL. Below the multicritical point the phase boundary splits into two parts, one separating the paramagnetic and spin glass phases and the other separating the ferromagnetic and spin glass phases. The duality transformation $x_{0} \leftrightarrow x_{0}^{*}$ exchanges these two phase boundaries; the uniqueness assumption of the transition point is not satisfied in this case below the multicritical point. The NL has a high symmetry since it satisfies $x_{1}=x_{2}$ as discussed in equation (34), which is a globally self-dual subvariety because $n$ is even, see equation (401). This means that the NL is mapped to itself by the duality transformation and we are allowed to identity the transition point on the NL (the multicritical point) with the fixed point, $x_{0}=x_{0}^{*}$, which indeed gives the exact location 


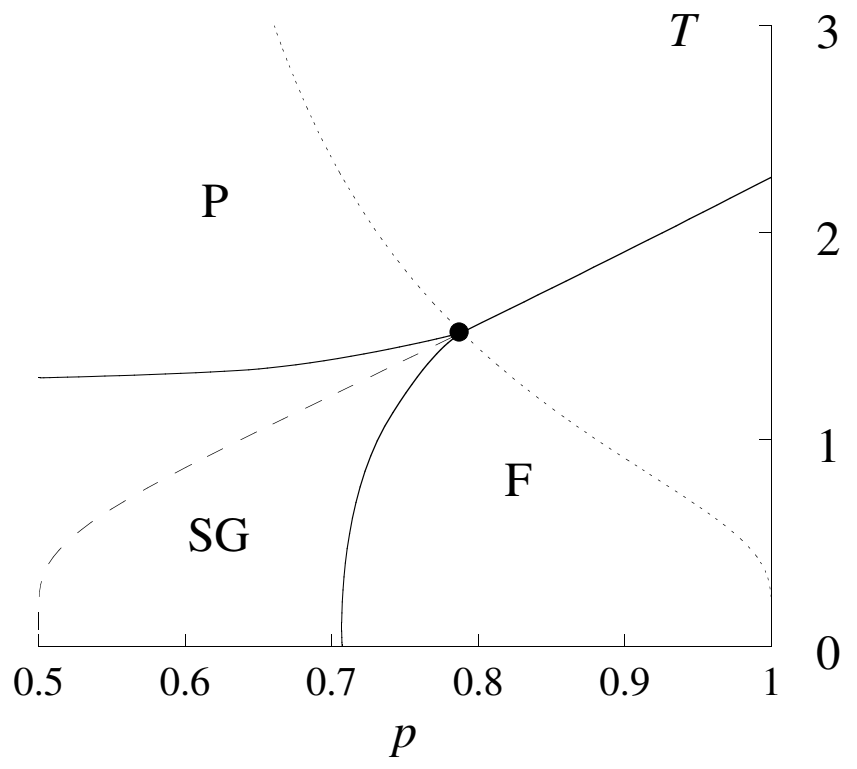

Figure 3. Phase diagram of the $\pm J$ Ising model with $n=2$. The condition $x_{0}=x_{0}^{*}$ gives the correct phase boundary (full curve) above the multicritical point but not below (shown dashed).

of the multicritical point. The lesson is that the condition $x_{0}=x_{0}^{*}$ does not always give the exact phase boundary but it may make sense to search for the multicritical point on the NL by investigating the intersection of the NL and the $x_{0}=x_{0}^{*}$ curve.

Another straightforward case is the general $2^{n}$-state standard scalar Potts model which has $u_{1}=u_{2}=u_{3}=\cdots=u_{n} \equiv u$ : The reduced partition function has then only a single variable $\mathcal{Z}_{n}(u)$, and we can identify the duality fixed point $u=u^{*}$ with the unique transition point. It is worth pointing out that the condition $x_{0}=x_{0}^{*}$ together with equation (82) automatically means that the values of $\mathcal{Z}_{n}$ on both sides are equal $\mathcal{Z}_{n}(u)=\mathcal{Z}_{n}\left(u^{*}\right)$ and consequently the arguments are equal $u=u^{*}$. Therefore the fixed point condition of the principal edge Boltzmann factor $x_{0}=x_{0}^{*}$ suffices to identify the transition point (assuming it is unique) in the highly symmetric case of the standard scalar Potts model. Note that, when $n=2$, the $\pm J$ Ising model on the NL coincides with the four-state standard scalar Potts model.

Let us next move on to the case of general $n$. The subvariety $\mathcal{N}$ defined as the set of points satisfying $u_{1}=u_{n}, u_{2}=u_{n-1}, u_{3}=u_{n-2}, \cdots$ is transformed into the dual $\mathcal{N}^{*}$ satisfying $u_{1}^{*}=u_{2}^{*}, u_{3}^{*}=u_{4}^{*}, \cdots$ as discussed in section 2.4 In addition, the subvariety $\mathcal{N}$ is globally self-dual $\mathcal{N}=\mathcal{N}^{*}\left(u_{1}^{*}=u_{n}^{*}, u_{2}^{*}=u_{n-1}^{*}, \cdots\right)$ when $n$ is even. The number of independent variables of $\mathcal{Z}_{n}$ is $[(n+1) / 2]$ in $\mathcal{N}$, and it is not possible to straightforwardly apply the argument developed above for the simple case of a single variable. If we further restrict ourselves to the NL of the $\pm J$ Ising model, we consider the one-dimensional curve in $\mathcal{N}$ representing the the condition (33), which does not necessarily coincide with its dual (except in the simplest cases of $n=1$ and 2). Therefore it is difficult to use duality 
arguments to locate the transition point for generic $n$ even if we restrict ourselves to the subvariety $\mathcal{N}$ (which satisfies a certain amount of symmetry) or its one-dimensional submanifold NL.

We nevertheless proceed by trying an ansatz that the fixed point condition of the principal component of the edge Boltzmann matrix $x_{0}=x_{0}^{*}$ may lead to the exact location of the transition point if we restrict ourselves to the NL in the subvariety $\mathcal{N}$. This ansatz is partly motivated by the above-mentioned example of the standard scalar Potts model, in which the condition $x_{0}=x_{0}^{*}$ was sufficient to find the exact transition point.

One may wish to try a different fixed point condition like $x_{1}=x_{1}^{*}$ to investigate the possible location of the transition point. A physical reason to choose $x_{0}=x_{0}^{*}$, not the other ones, is that this edge Boltzmann factor $x_{0}$ is special in the sense that it is for the all-parallel spin configuration, and the expression of duality in equation (82), which singles out $x_{0}$ as a special variable, means to measure the energy relative to the perfectly ferromagnetic state. This is natural when we discuss the properties of the system on the NL which starts from the perfectly ferromagnetic ground state $(p=1, T=0)$ and terminates in the perfectly random high-temperature limit $\left(p=\frac{1}{2}, T \rightarrow \infty\right)$ and the multicritical point on this line separates the ferromagnetic and paramagnetic phases [4]. Another reason is that, if we try another condition, $x_{1}=x_{1}^{*}$ for example, the result does not satisfy the rigorous inequality (152). This and other related points will be discussed in more detail later.

The present ansatz $x_{0}=x_{0}^{*}$ to locate the multicritical point on the NL is equivalent to the one given in [16].

The condition $x_{0}=x_{0}^{*}$ is written explicitly for the $\pm J$ Ising model as, using equations (8) and (20),

$$
p e^{n K}+(1-p) e^{-n K}=2^{-n / 2}\left(e^{K}+e^{-K}\right)^{n} .
$$

This relation, in conjunction with the NL condition (33), yields

$$
e^{(n+1) K}+e^{-(n+1) K}=2^{-n / 2}\left(e^{K}+e^{-K}\right)^{n+1} .
$$

This equation, derived from $x_{0}=x_{0}^{*}$ plus the NL condition, gives the exact transition point (multicritical point) in the cases of $n=1$ and 2 as already discussed. When $n=3$, the curve given by equation (83) is drawn in figure 4 (marked 0) together with the curves coming from three other conditions $x_{1}=x_{1}^{*}, x_{2}=x_{2}^{*}$ and $x_{3}=x_{3}^{*}$ marked 1, 2 and 3, respectively. Apparently the intersections of the NL (dotted curve) and the four curves corresponding to $x_{0}=x_{0}^{*}, x_{1}=x_{1}^{*}, x_{2}=x_{2}^{*}, x_{3}=x_{3}^{*}$ can be candidates of the transition point. To check these possibilities, let us note that equation (84) coming from $x_{0}=x_{0}^{*}$ gives $T_{\mathrm{c}}^{(3)}=1.65858$ as a candidate of the multicritical point for $n=3$. Similarly, $T_{\mathrm{c}}^{(4)}=1.75717$ for $n=4$, and $T_{\mathrm{c}}^{(5)}=1.82955$ for $n=5$ if we consistently use equation (84). When $n=1$ and $n=2$, the same equation leads to $T_{\mathrm{c}}^{(1)}=1.30841$ and $T_{\mathrm{c}}^{(2)}=1.51865$, both of which agree with the exact solution as they should. These conjectured values of $T_{\mathrm{c}}^{(n)}$ from the condition $x_{0}=x_{0}^{*}$ plus the NL all satisfy the rigorous 


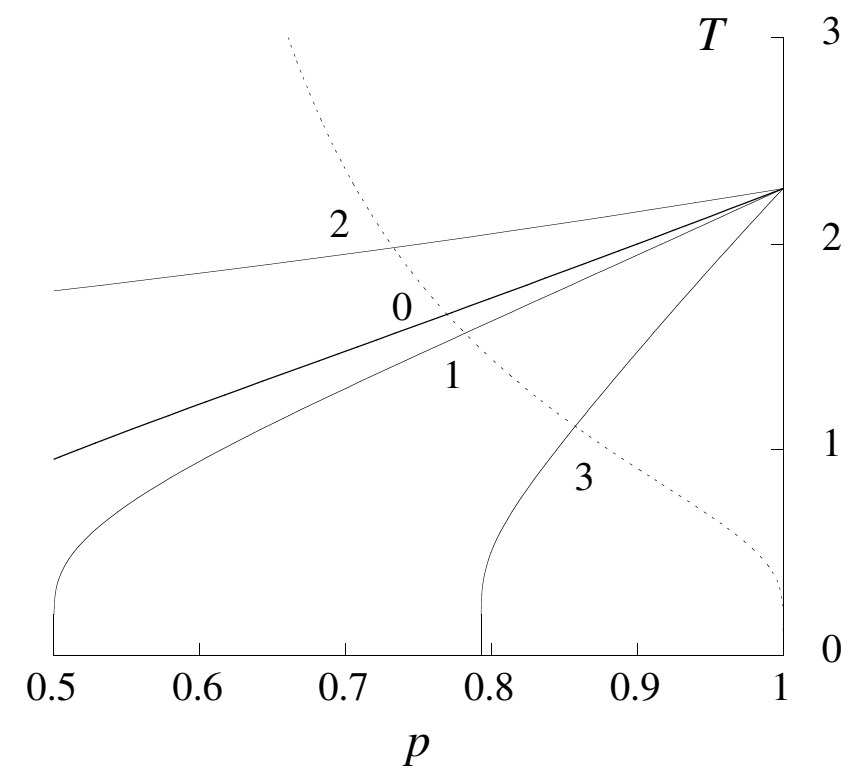

Figure 4. The curves representing $x_{0}=x_{0}^{*}, x_{1}=x_{1}^{*}, x_{2}=x_{2}^{*}$ and $x_{3}=x_{3}^{*}$ marked 0 , $1,2,3$, respectively, for the $n=3$ model.

inequality (52):

$$
T_{\mathrm{c}}^{(1)}<T_{\mathrm{c}}^{(2)}<T_{\mathrm{c}}^{(3)}<T_{\mathrm{c}}^{(4)}<T_{\mathrm{c}}^{(5)} .
$$

If we instead assume that $x_{1}=x_{1}^{*}$ together with the NL condition (33) may give the multicritical point, the same inequality is violated at $n=4$ because, then, $T_{\mathrm{c}}^{(3)}=1.56655$ and $T_{\mathrm{c}}^{(4)}=1.51865$. The same is true for the other $x_{k}=x_{k}^{*}(k \geq 2)$. Thus $x_{0}=x_{0}^{*}$ is the only possibility consistent with the inequality if we are to choose the multicritical point among the intersections of $x_{k}=x_{k}^{*}(k=0,1, \cdots, n)$ and the NL.

It should be of some interest to discuss the limit $n \rightarrow \infty$ because the problem can be solved exactly. The $n$-replicated partition function for arbitrary $n$ is

$$
Z_{n}=\left[Z(K)^{n}\right]_{\mathrm{av}}=\left[e^{-n \beta N f(K)}\right]_{\mathrm{av}} .
$$

If we take the limit $n \rightarrow \infty$ with $N$ (the number of sites) kept large but finite, the number of terms in the above configurational average remains finite, and the value of this equation is dominated by the term with the smallest value of $f(K)$. The smallest value of the free energy is expected to be given by the perfectly ferromagnetic bond configuration (and its gauge equivalents), and therefore we have

$$
Z_{n} \approx e^{-\beta_{p} N f_{0}\left(K_{p}\right)} e^{-n \beta N f_{0}(K)},
$$

where $f_{0}$ is the free energy of the non-random ferromagnetic Ising model. The first factor on the right-hand side is the probability weight of the non-random configuration and its gauge equivalents (proportional to $Z\left(K_{p}\right)$; see section 2.8). The system then has a unique critical point at the non-random critical point $K=K_{\mathrm{c}}^{\mathrm{F}}$ as well as at $K_{p}=K_{\mathrm{c}}^{\mathrm{F}}$ (the latter being equivalent to $p_{\mathrm{c}}=\frac{\sqrt{2}}{2}$ ). The phase boundaries are two lines 
representing these two critical parameters. On the other hand, the condition $x_{0}=x_{0}^{*}$ gives in the limit $n \rightarrow \infty$

$$
e^{-2 K}=\sqrt{2}-1
$$

which agrees with the above analysis giving $K=K_{\mathrm{c}}^{\mathrm{F}}$. Thus the conjecture that the multicritical point is given by the intersection of $x_{0}=x_{0}^{*}$ and the NL is correct in this limit $n \rightarrow \infty$. It is interesting to note that for $n \rightarrow \infty$ all the conditions $x_{k}=x_{k}^{*}$ with $k$ finite lead to the same conclusion. However, for finite $n$, the result depends on $k$, and the $k=0$ condition is the only one consistent with the inequality (52).

\subsection{Numerical evidence for $n=3$}

We have carried out an extensive Monte Carlo simulation of the $n=3 \pm J$ Ising model on the NL on the square lattice to check the above-mentioned possible value of the multicritical point $T_{\mathrm{c}}^{(3)}=1.65858$. A preliminary scan of the energy showed that the transition is very likely to be of first order since clear hysteresis has been found. This is natural because the system has eight $\left(=2^{3}\right)$ degrees of freedom at each site and is not very far from the eight-state standard scalar Potts model (which has a first-order transition) because of the enhanced symmetry $x_{1}=x_{3}$ on the NL (a little short of the high symmetry $x_{1}=x_{2}=x_{3}$ of the standard scalar Potts model).

We have therefore used the non-equilibrium relaxation method ([17, 18] and references therein), which allows us to simulate very large systems in its initial relaxation stage to investigate equilibrium properties. Another reason to use the non-equilibrium relaxation method is that it allows us to identify the first-order transition point without evaluating the free energy [17. The results for the relaxation of magnetization are drawn in figure 5 for the temperature range from $T=1.650$ (top curve) to $T=1.665$ (bottom curve) with linear system size $L=8000$, averaged over several samples, under mixed phase initialization appropriate for first-order transition [17]. We have confirmed by comparison with smaller systems (and larger systems $(L=12000)$ in some limited cases) that this size $L=8000$ is sufficiently large to investigate the properties of infinite-size systems at least to the time steps indicated in figure 5 (2000 steps). From the positive slope and upward curvature of magnetization after about 1000 Monte Carlo steps for $T=1.655$, we conclude that this temperature is in the ferromagnetic phase due to the prescription of the non-equilibrium relation method [17]. Similarly the system is judged to lie in the paramagnetic phase at $T=1.665$ from the negative slope and downward curvature. Our conclusion is $T_{\mathrm{c}}^{(3)}=1.660(5)$, which is consistent with the above-mentioned conjecture 1.65858. The values from other fixed point conditions are completely ruled out, 1.56655 (from $\left.x_{1}=x_{1}^{*}\right), 1.98207\left(x_{2}=x_{2}^{*}\right)$ and $1.11466\left(x_{3}=x_{3}^{*}\right)$.

\subsection{Quenched limit}

There have been a number of numerical studies of quenched systems $(n \rightarrow 0)$ to find the location of multicritical point on the square lattice. If we take the limit $n \rightarrow 0$ 


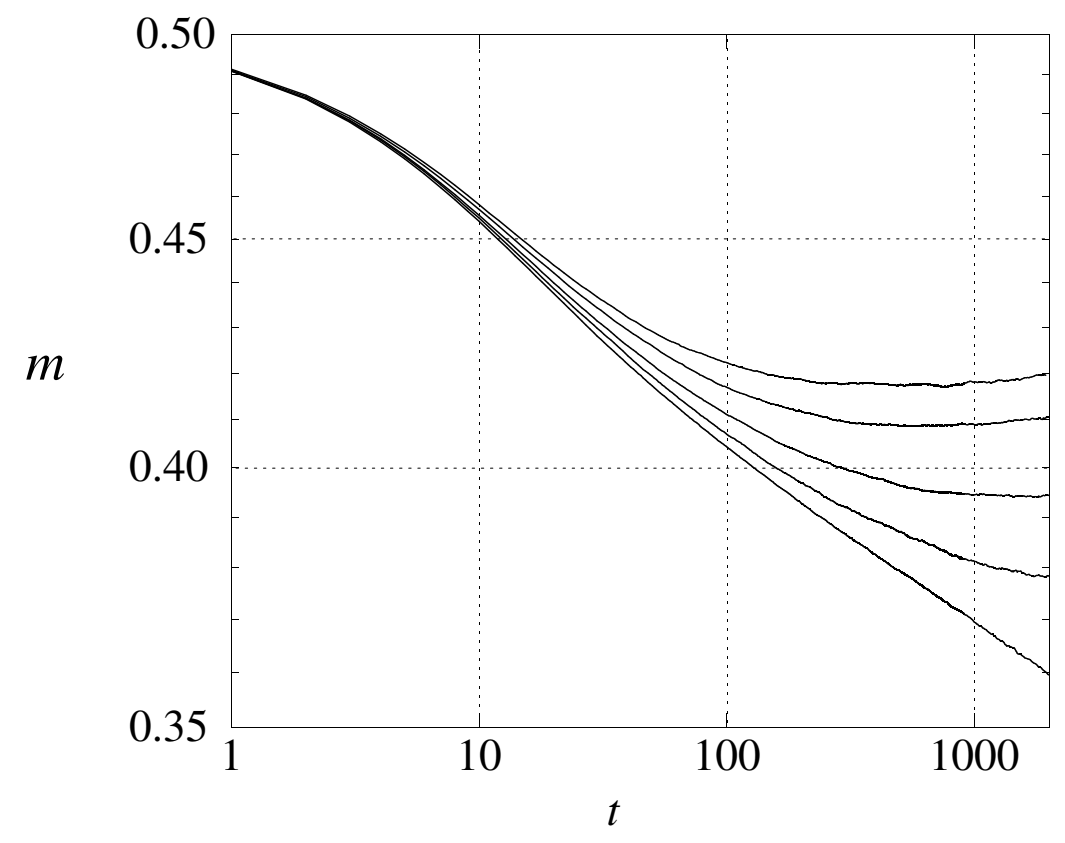

Figure 5. Non-equilibrium relaxation of magnetization with mixed phase initialization for the $n=3 \pm J$ Ising model on the NL on the square lattice. The temperatures are $1.650,1.655,1.660,1.663,1.665$ from top to bottom.

in equation (84), the formula rewritten in terms of $p$ acquires a simple and appealing expression suggesting that exactly half of the entropy of bond distribution is exhausted at $p_{\mathrm{c}}$ :

$$
-p_{\mathrm{c}} \ln p_{\mathrm{c}}-\left(1-p_{\mathrm{c}}\right) \ln \left(1-p_{\mathrm{c}}\right)=\frac{\ln 2}{2} .
$$

Numerically this equation gives $p_{\mathrm{c}}=0.88997$, which is compared very favourably with numerical results, among which $0.8894(9)$ is the most recent and extensive one [18], as well as 0.8905(5) [19, 0.886(3) [20, 0.8906(2) [21] and 0.8907(2) [22].

Interestingly, the curve (83) coming from $x_{0}=x_{0}^{*}$ is the only one with an intersection with the NL in the quenched limit. All the other curves $x_{k}=x_{k}^{*}(k \geq 1)$ do not cross the NL as depicted in figure 6.

If we further apply the condition $x_{0}=x_{0}^{*}$ to a model with continuous distribution of coupling as in equation (69), we find in the limit $n \rightarrow 0$

$$
\int d u P(u) \ln \left(1+e^{-2 \beta u}\right)=\frac{\ln 2}{2} .
$$

This expression already appeared in [23]. Under the NL condition (711), this equation reads

$$
\int d u e^{\beta u} F\left(u^{2}\right) \ln \left(1+e^{-\beta u}\right)=\frac{\ln 2}{2},
$$

which gives for the Gaussian model $J_{0} / J=1.02177$. Numerical results are consistent with this value, 1.00(2) (Y Ozeki et al, private communication). 


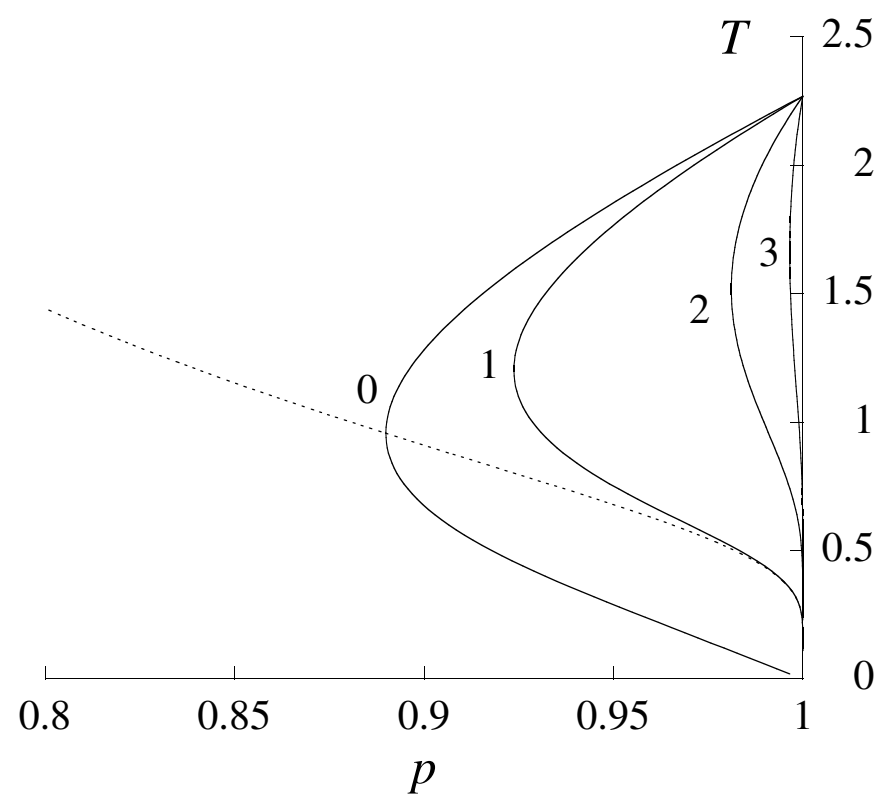

Figure 6. The curves representing fixed point conditions $x_{0}=x_{0}^{*}, x_{1}=x_{1}^{*}, x_{2}=x_{2}^{*}$ and $x_{3}=x_{3}^{*}$ marked $0,1,2$ and 3 , respectively, in the quenched limit. The NL is shown dotted.

For the random chiral Potts model with

$$
\begin{aligned}
& V(0)=K, V(1)=V(2)=\cdots=V(q-1)=0 \\
& p_{0}=1-(q-1) p, p_{1}=p_{2}=\cdots p_{q-1}=p
\end{aligned}
$$

under the NL condition 24

$$
p=\frac{1}{e^{K}+q-1},
$$

the conjectured multicritical point coming from $x_{0}=x_{0}^{*}$ is in the quenched limit, using equations (74) and (75),

$$
-\left(1-(q-1) p_{c}\right) \ln \left(1-(q-1) p_{c}\right)-(q-1) p_{c} \ln p_{c}=\frac{\ln q}{2} .
$$

Note that the model with equation (93) reduces to the non-random case in the limit $p \rightarrow 0$ in contrast to our previous convention of $p \rightarrow 1$; the reason is to avoid confusion when we compare our conjecture with the numerical investigation which suggests $p_{\mathrm{c}}$ between 0.079 and 0.080 for $q=3$ [25]. Equation (95) yields $p_{\mathrm{c}}=0.079731$ for $q=3$. We therefore conclude that the condition $x_{0}=x_{0}^{*}$ has been confirmed to give the correct transition point on the NL within numerical accuracies for all available cases.

\section{Summary and Discussions}

We have derived symmetries of the models of the two-dimensional spin glass. In particular, invariances of the replicated partition function $Z_{n}$ under exchanges of edge 
Boltzmann factors and duality have been proved. Also discussed is the invariance of the structure of the edge Boltzmann matrix by inversions. These properties hold for generic values of edge Boltzmann factors, not just for the replicated $\pm J$ Ising model. The dual Boltzmann factor of the $\pm J$ Ising model has an interesting expression in terms of dual spin variables. Griffiths inequalities apply to this expression, leading to monotonicity of phase boundaries, equivalent to monotonicity of the location of multicritical point as a function of $n$.

Complexities under inversions have been investigated and a remarkable reduction of complexities (enhanced symmetry) has been observed in the subvariety $\mathcal{N}$. This result suggests that the behaviour of the system is simpler in this subvariety than at generic points even if the problem is not (generically) integrable because the exponential growth excludes integrability.

Conjecture on the exact location of the multicritical point on the NL has been presented based on the duality and symmetry arguments. Reasons have been explained that the intersection of the curve $x_{0}=x_{0}^{*}$ and the NL is the only plausible candidate of the multicritical point among a set of similar conjectures $\left(x_{k}=x_{k}^{*}\right.$ plus the NL). Numerical results are in very good agreement with this conjecture. Nevertheless, recalling the situation already encountered for the analysis of the phase diagram of the isotropic three-state chiral Potts model [27, 28, where the critical manifold is numerically extremely close to the self-dual condition $x_{0}^{*}=x_{0}$ but is possibly mathematically different (in contrast with the situation encountered in the symmetric Ashkin-Teller model), one cannot discard the possibility that, restricted to the NL, the transition point could be numerically extremely close to the intersection with $x_{0}^{*}=x_{0}$ but, actually, mathematically different. These points need to be further investigated carefully.

It may be useful to consider the possibility that the condition $x_{0}=x_{0}^{*}$ could be a very good approximation of the transition manifold away from the intersection with $\mathcal{N}$ but in the high temperature part of the phase boundary as in figure 3 at least for random ferromagnetic spin systems. For the $q$-state random standard ferromagnetic Potts model (the random version of the ferromagnetic scalar Potts model), the condition $x_{0}=x_{0}^{*}$ in the quenched limit is

$$
\int d \mu\left(e^{K}\right) \ln \left(\frac{e^{K}+(q-1)}{e^{K}}\right)=\frac{1}{2} \ln q,
$$

where the positive interaction $K$ is assumed to be distributed with measure $d \mu\left(e^{K}\right)$. This is a Potts-generalization of equation (90). Investigation of the consequences of this equation is going on.

Another interesting observation is that the formula (84) gives $K$ as a function of $n$ which diverges as $n$ approaches -1 . It is not obvious at all that we can apply this formula to such a limit, but if we do so, then the divergence of $K$ implies that the transition point on the NL is $T_{\mathrm{c}}^{(n)} \rightarrow 0$ as $n \rightarrow-1$. If we use the relation between $n$ and $n+1$ in equation (58), we find that the transition point of the $n \rightarrow 0$ (quenched) system vanishes at $p=\frac{1}{2}$. Although we should be very careful to apply our results to 
such a limit of negative $n$, this last result is reasonable and interesting in its own right because it supports the usual consensus that the two-dimensional Ising spin glass does not have a finite-temperature phase transition when the distribution of bond randomness is symmetric. More efforts should be devoted to the investigation of this problem.

Let us also comment here on the equivalence between the $n$-replicated $3 d$ random lattice gauge theory and its dual, the $n$-replicated Ising spin ferromagnet with edge Boltzmann factor described in section 2.6. All the arguments sections 2.6] and 2.7 apply to such a case. In particular the bound on the critical probability like (54) results: $p_{\mathrm{c}}^{(0)}(\mathrm{MCP}) \geq 0.9005$. Numerically it is $0.97[26] \sharp$.

We have seen that the replica analysis naturally yields to consider a class of remarkable (non-random) lattice spin models, defined by equation (6), which are highly structured and for which extensive symmetry analysis can be performed exactly. Recalling Domany's $\left(N_{\alpha}, N_{\beta}\right)$ terminology [29], these models correspond to highly symmetric specific $\left(N_{2}, \cdots, N_{2}\right)$ models and, more generally $\left(N_{q}, \cdots, N_{q}\right)$ models. These singled-out classes of lattice spin edge models provide a very powerful tool of analysis for many spin-glass problems and are also worth studying per se.

One should note that many of the exact calculations, displayed in this paper, are still valid when the distribution of the coupling constants is not of the $\pm J$ type or some continuous distribution like equation (71) but, for instance, a two-delta-peaks $\left(J_{1}, J_{2}\right)$ distribution, or, even, a totally general distribution : We only need to get an effective Boltzmann matrix of the hierarchical form (6). One should also underline that these models can straightforwardly be generalized to chiral Potts models (see Appendix B), and also to spin models without any Wu-Wang duality (like the Ising model with a magnetic field, see Appendix B ; the non-linear inversion relations $I$ and $J$ taking the place of the linear duality transformation $D$ ), providing room for many new exact results on spin-glass problems.

\section{Acknowledgments}

The work of HN was supported by the Grant-in-Aid for Scientific Research by the Ministry of Education.

\section{Appendix A.}

In this appendix we outline the proof of equation (40) assuming equation (34) and the symmetry (24) under the operation $M$. According to the duality relation (15) for even $n=2 q$, the expression of $x_{m}^{*}$, after applying the operation $M$, reads

$$
2^{q} x_{m}^{*}=\sum_{k=0}^{q} D_{m}^{2 k} x_{2 k}+\sum_{k=1}^{q} D_{m}^{2 k-1} x_{2 k-1}
$$

$\sharp$ This value is for the $T=0$ transition point, not for the multicritical point, but is likely to be close to the latter. 
Symmetry, complexity and multicritical point of the two-dimensional spin glass

$$
\begin{aligned}
\rightarrow & \sum_{k=0}^{q} D_{m}^{2 k} x_{2 k}+\sum_{k=1}^{q} D_{m}^{2 k-1} x_{2 q-2 k+1} \\
= & D_{m}^{0} x_{0}+D_{m}^{2} x_{2}+D_{m}^{4} x_{4}+\cdots+D_{m}^{2 q} x_{2 q} \\
& +D_{m}^{1} x_{2 q-1}+D_{m}^{3} x_{2 q-3}+\cdots+D_{m}^{2 q-1} x_{1} .
\end{aligned}
$$

We then impose the condition (34) to find

$$
2^{q} x_{m}^{*}=D_{m}^{0} x_{0}+\left(D_{m}^{1}+D_{m}^{2}\right) x_{2}+\left(D_{m}^{3}+D_{m}^{4}\right) x_{4}+\cdots+\left(D_{m}^{2 q-1}+D_{m}^{2 q}\right) x_{2 q} .
$$

Thus, in order to show $x_{m}^{*}=x_{2 q-m+1}^{*}$, it suffices to derive

$$
D_{m}^{2 k-1}(2 q)+D_{m}^{2 k}(2 q)=D_{2 q-m+1}^{2 k-1}(2 q)+D_{2 q-m+1}^{2 k}(2 q),
$$

where we have written the $n(=2 q)$-dependence explicitly. This equation can be proved by induction with respect to $q$.

The following relation will be useful for the proof:

$$
D_{m+1}^{k}(n)+D_{m+1}^{k-1}(n)=D_{m}^{k}(n)-D_{m}^{k-1}(n)
$$

which is derived by replacing $m$ in equation (16) with $m+1$ (which amounts to multiplying both sides by $(1-t) /(1+t))$.

It is easy to check explicitly using equation (17) that the target relation (A.3) is valid for small $q$ with any $m$ and $k$. Let us then assume that this equation holds for $q$ with any $m$ and $k$ and show that the same is true for $q+1$. The left-hand side of equation (A.3) with $2 q$ replaced by $2 q+2$ can be reduced to an expression with $2 q$ using the recursion relation (39) twice,

$$
\begin{aligned}
& D_{m}^{2 k-1}(2 q+2)+D_{m}^{2 k}(2 q+2) \\
= & D_{m}^{2 k}(2 q)+D_{m}^{2 k-1}(2 q)+2\left(D_{m}^{2 k-1}(2 q)+D_{m}^{2 k-2}(2 q)\right) \\
& +D_{m}^{2 k-2}(2 q)+D_{m}^{2 k-3}(2 q) .
\end{aligned}
$$

Application of the other recursion relation (A.4) to each pair of terms on the right-hand side of the above equation yields

$$
\begin{aligned}
& D_{m}^{2 k-1}(2 q+2)+D_{m}^{2 k}(2 q+2) \\
= & D_{m-1}^{2 k}(2 q)+D_{m-1}^{2 k-1}(2 q)-D_{m-1}^{2 k-2}(2 q)-D_{m-1}^{2 k-3}(2 q) .
\end{aligned}
$$

This is our expression for the left-hand side of equation (A.3) with $q \rightarrow q+1$. The right-hand side of equation (A.3) with $2 q$ replaced by $2 q+2$ can also be rewritten by the recursions (39) and (A.4) to reach a similar expression

$$
\begin{aligned}
& D_{2 q+2-m+1}^{2 k-1}(2 q+2)+D_{2 q+2-m+1}^{2 k}(2 q+2) \\
= & D_{2 q-m+2}^{2 k}(2 q)+D_{2 q-m+2}^{2 k-1}(2 q)-D_{2 q-m+2}^{2 k-2}(2 q)-D_{2 q-m+2}^{2 k-3}(2 q) .
\end{aligned}
$$

This equation is equal to equation (A.6) by the starting assumption of induction, which completes the proof. 
Symmetry, complexity and multicritical point of the two-dimensional spin glass

\section{Appendix B.}

The results on the Ising model generalize straightforwardly to $q$-state models. Let us consider, for instance, the three-state chiral Potts model corresponding to a $3 \times 3$ cyclic edge Boltzmann matrix $\dagger \dagger$. For an arbitrary number $n$ of replicas, the previous hierarchical scheme generalizes straightforwardly. If one denotes by $A_{n}$ the $q^{n} \times q^{n}$ effective edge Boltzmann matrices corresponding to $\left[Z^{n}\right]_{\text {av }}$ they can be obtained by the following recursion :

$$
A_{n} \rightarrow A_{n+1}=\left[\begin{array}{ccc}
A_{n} & B_{n} & C_{n} \\
C_{n} & A_{n} & B_{n} \\
B_{n} & C_{n} & A_{n}
\end{array}\right]
$$

where

$$
A_{1}=\left[\begin{array}{ccc}
x_{0,0} & x_{0,1} & x_{1,0} \\
x_{1,0} & x_{0,0} & x_{0,1} \\
x_{0,1} & x_{1,0} & x_{0,0}
\end{array}\right]
$$

and

$$
B_{n}=A_{n}\left(x_{m, p} \rightarrow x_{m, p+1}\right), \quad C_{n}=A_{n}\left(x_{m, p} \rightarrow x_{m+1, p}\right) .
$$

Note that the number of homogeneous parameters necessary to describe the pattern (B.1) grows quadratically with the number of replicas like $(n+2) \cdot(n+1) / 2$ (this number is 3 for $n=1,6$ for $n=2,10$ for $n=3,15$ for $n=4,21$ for $n=5$, ...). The family of $3^{n} \times 3^{n}$ models we have to study is thus a little more complicated (as well as the equivalent of the subvarieties $\mathcal{N}(34)$ ). This is in contrast with the $q=2$ Ising model without magnetic field, analysed in the text, for which the number of homogeneous parameters $x_{k}$ grows linearly with $n$ (this number is $n+1$ ). More generally, for a cyclic $q \times q$ matrix, the number of homogeneous parameters necessary to describe a pattern generalizing (B.1) will be the number of partition of $n$ into $q$ natural integers $k_{i}: k_{1}=k_{2}+k_{3}+\cdots+k_{q}=n$ with $k_{i}=0,1, \cdots n$. The duality transformation on these models (B.1) is inherited from the duality transformation on the three-state chiral Potts model associated with cyclic $3 \times 3$ matrices.

Let us consider a simple example of spin edge model with no duality transformation, namely the Ising model with a magnetic field. The effective Boltzmann matrices can be obtained similarly by a hierarchical scheme generalizing (6) :

$$
A_{n} \rightarrow A_{n+1}=\left[\begin{array}{cc}
A_{n} & B_{n} \\
B_{n} & C_{n}
\end{array}\right]
$$

where

$$
A_{1}=\left[\begin{array}{ll}
x_{0,0} & x_{1,0} \\
x_{1,0} & x_{0,1}
\end{array}\right]
$$

††That is the chiral Potts model studied by Baxter, Perk and Au-Yang [30]. 
and

$$
B_{n}=A_{n}\left(x_{m, p} \rightarrow x_{m+1, p}\right), \quad C_{n}=A_{n}\left(x_{m, p} \rightarrow x_{m, p+1}\right) .
$$

Again the number of homogeneous parameters necessary to describe the pattern (B.3) grows quadratically with the number of replicas like $(n+2) \cdot(n+1) / 2$. In this last case, we do not have a duality transformation $D$ anymore: The inversion relations $I$ and $J$ take the place of the linear duality transformation $D$. However, the inverse of matrices given by (B.3) are not matrices of the same form (B.3). One thus needs to consider slightly more general matrices than $(\mathrm{B} .3)$ in order to be able to use the non-linear symmetry $I$. Details will be given elsewhere.

\section{References}

[1] Mézard M, Parisi G and Virasoro M A 1987 Spin Glass Theory and Beyond (World Scientific: Singapore)

[2] Young A P (Ed) 1997 Spin Glasses and Random Fields (World Scientific: Singapore)

[3] Nishimori H 1981 Prog. Theor. Phys. 661169

[4] Nishimori H 2001 Statistical Physics of Spin Glasses and Information Processing: An Introduction (Oxford University Press: Oxford)

[5] Anglès d'Auriac J -Ch, Maillard J-M and Viallet C M 2002 J. Phys. A: Math. Gen. 359251

[6] Wu F Y and Wang Y K 1976 J. Math. Phys. 17439

[7] Georges A, Hansel D, Le Doussal P and Maillard J -M 1987 J. Physique 481

[8] Meyer H, Anglès d'Auriac J -Ch, Maillard J -M and Rollet G 1994 Physica A 208223

[9] Boukraa S, Maillard J -M 2001 J. Stat. Phys. 102641

[10] Nishimori H 2002 J. Phys. A: Math. Gen. 359541

[11] Griffiths R B 1972 in Phase Transitions and Critical Phenomena, Vol. 1, Eds C Domb and M S Green, (Academic Press: London)

[12] Nishimori H 1986 J. Phys. Soc. Japan 553305

[13] Abarenkova N, Anglès d'Auriac J-Ch, Boukraa S and Maillard J -M 1999 Physica D 13027

[14] Abarenkova N, Anglès d'Auriac J-Ch, Boukraa S, Hassani S and Maillard J -M 1999 Physica A 264264

[15] GNU Multiprecision; see http://www.swox.com/gmp/

[16] Nishimori H and Nemoto K 2002 J. Phys. Soc. Japan 711198

[17] Ozeki Y, Kasono K, Ito N and Miyashita S 2003 Physica A 321271

[18] Ito N, and Ozeki Y 2003 Physica A 321262

[19] Aarao Reis F D A, de Queiroz S L A and dos Santos R R 1999 Phys. Rev. B 606740

[20] Singh R R P and Adler J 1996 Phys. Rev. B 54364.

[21] Honecker A, Picco M and Pujol P 2001 Phys. Rev. Lett. 87047201

[22] Merz F and Chalker J T 2002 Phys. Rev. B 65054425

[23] Nishimori H 1979 J. Phys. C: Solid State Phys. 12 L905

[24] Nishimori H and Stephen M J 1983 Phys. Rev. B 275644

[25] Jacobsen J L and Picco M 2002 Phys. Rev. E 65026113

[26] Wang C, Harrington J and Preskill J 2003 Ann. Phys., NY 30331

[27] Anglès d' Auriac J-Ch, Maillard J-M and Wu F Y 1991 Physica A 177114

[28] Anglès d' Auriac J-Ch, Maillard J-M and Wu F Y 1991 Physica A 179496

[29] Domany E and Riedel E K 1978 Phys. Rev. Lett. 40561

[30] Baxter R J, Perk J H H and Au-Yang H 1988 Phys. Lett. A 128138 